\title{
Characteristics of Unsteady Total Pressure Distortion for a Complex Aero-engine Intake Duct
}

\author{
Geoffrey Tanguy ${ }^{1}$, David G. MacManus ${ }^{2}$ \\ Propulsion Engineering Centre, School of Aerospace, Transport and Manufacturing, Cranfield University, MK43 OAL, UK \\ Eric Garnier ${ }^{3}$ \\ ONERA, French Aerospace lab, 8 Rue des Vertugadin, Meudon 92190, France \\ Peter G. Martin ${ }^{4}$ \\ Defence Science and Technology Laboratory, Portsdown West, Fareham, UK, PO17 6AD
}

\begin{abstract}
Some types of aero-engine intake systems are susceptible to the generation of secondary flows with high levels of total pressure fluctuations. The resulting peak distortion events may exceed the tolerance level of a given engine, leading to handling problems or to compressor surge. Previous work used distortion descriptors for the assessment of intake-engine compatibility to characterise modestly curved intakes where most of the self-generated time-dependent distortion was typically found to be dominated by stochastic events. This work investigates the time-dependent total pressure distortion at the exit of two high off-set diffusing S-duct intakes with the aim of establishing whether this classical approach, or similar, could be applied in these instances. The assessment of joint probability maps for time dependent radial and circumferential distortion metrics demonstrated that local ring-based distortion descriptors are more appropriate to characterise peak events. Extreme Value Theory (EVT) was applied to predict the peak distortion levels that could occur for a test time beyond the experimental data set available. Systematic assessments of model sensitivities to the de-clustering frequency, the number of exceedances and sample time length were used to extend the EVT application to local distortion descriptors and to provide guidelines on its usage.
\end{abstract}

Keywords: Unsteady total pressure distortion, S-duct intake, Extreme Value theory

\section{Nomenclature}

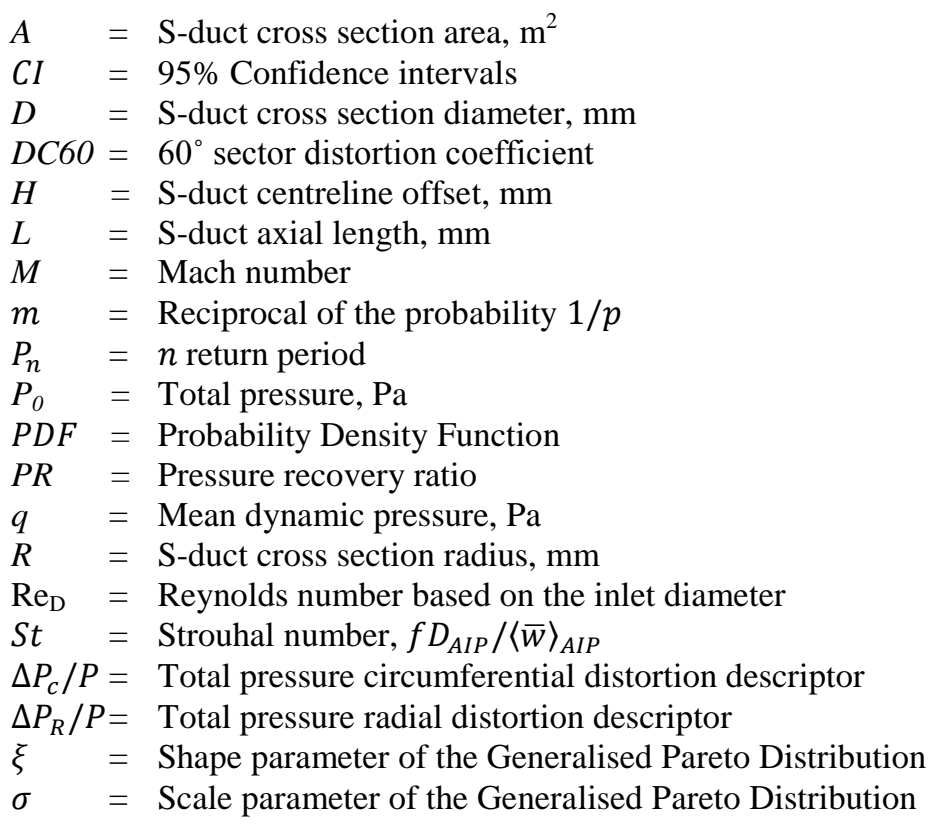

\section{Operators}

(·) $=$ time averaged

\footnotetext{
${ }^{1}$ PhD Student, Propulsion Engineering Centre, Building 52, Cranfield University

${ }^{2}$ Senior Lecturer, Propulsion Engineering Centre, Building 52, Cranfield University

${ }^{3}$ Research Scientist, Applied Aerodynamics Department, 8 Rue des Vertugadins, Meudon

${ }^{4}$ Research Scientist, Dstl Platform Systems, i-Sat C, Room D119, Grenville East Court, Portsdown West
} 
$\overline{-} \quad=$ area weighted averaged

$\sigma \quad=$ standard deviation

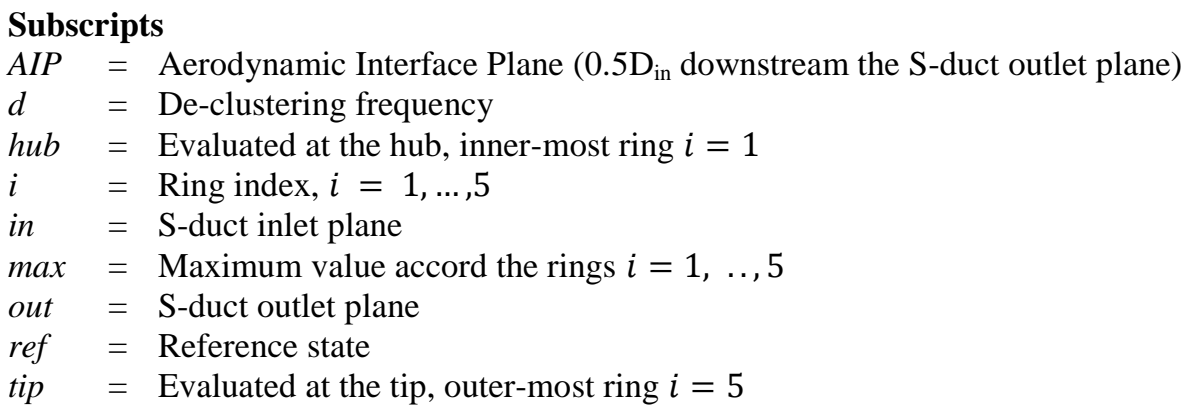

\section{Introduction}

Time variant total-pressure distortion in air intakes first began to be recognized as a significant performance limiting consideration for aircraft engine installations in the 1960s [1]. Issues encountered late in the development cycle of a number of aircraft [2,3] led to a significant effort to characterize such intake flows as well as to understand engine response. As dynamic instrumentation capability was gradually improved, for both in-flight and wind tunnel settings, a wide range of flow distortion characteristics were identified [4]. Flow distortion can arise from a range of aerodynamic aspects such as of boundary layer ingestion, lip separation, shock induced separation and secondary internal flows. As a consequence, large unsteady perturbations of complex total pressure and swirl distortion flow fields are presented to the engine system. The adverse effect on engine performance and operability can be characterized by stall cells within the first stages of the compression system [5] and in the worst case can lead to engine surge [6]. Furthermore, discrete distortion regions can strongly affect the blade loading, mechanical vibration and fatigue life [7].

The effect of steady state total pressure distortion has received significant attention over the years with the development of distortion indices to evaluate radial and circumferential perturbations at an Aerodynamic Interface Plane (AIP) ahead of the engine fan face. Those indices were developed in order to correlate the flow distortion with the loss in surge margin for a give compressor. Although none of the distortion descriptors is universally used, the Society of Automotive Engineers (SAE) [4] developed a series of indices to characterize the intensity of the loss in total pressure and the shape assimilated to the distortion region at the AIP.

Fully embedded propulsion systems usually have complex convoluted ducts in which unsteady flow distortion arises from local flow separations and strong secondary flows. Although the time averaged AIP flow field statistics for such complex intakes provide an indication of the distortion level for a given operating condition, the complex interaction between secondary flows and separation regions can generate correlated features that are obscured by the overall variations of the distortion [8,9]. It is well established that steady distortion contributes to the reduction of surge margin for a given propulsion system [4].

Recent experimental work investigated the effect of total pressure distortion for an aero-engine [10]. A linear relationship was demonstrated between the level of total pressure loss at the AIP and the reduction in surge margin for the low pressure compressor. A similar trend was also noted with the Circumferential Distortion Index (CDI) and the $60^{\circ}$ based Circumferential Distortion descriptor (DC60). However the flow distortion within the S-duct is highly unsteady with large deviations from the mean flow [8,11]. The effect of the dynamic component of the distortion and its associated peak value has been known to be responsible for the rise of instabilities within the compressor even though the mean levels were within the acceptable operating limits [6]. It has also been shown that engine response is sensitive to the frequency content of the time variant distortion pattern [12] especially when it is within a few percent of the rotor shaft speed.

The dynamic aspect of the distortion was commonly treated by the use of a probabilistic approach to predict the most likely peak instantaneous distortion level from a limited measurement record. This approach may be used to describe how this level would vary with the amount of time spent at the corresponding flight condition [1,13]. Borg [14] and Melick et al. [15], developed a way of synthesizing distortion data from limited root mean squared (RMS) pressure measurements, which could greatly reduce instrumentation requirements in early-stage concept screening. This would also, much later, provide a useful basis for deriving timevariant distortion data from Computational Fluid Dynamic (CFD) simulations [16]. These approaches were subsequently extended by Sedlock [17] to estimate the most probable maximum level of RMS total pressure at the AIP. All of these methods required that pressure measurements at the engine face were temporally uncorrelated, and it was recognized from the outset that, where this was not the case, a deterministic empirical approach would be required. The Extreme Value Theory (EVT) initially developed by Jacocks [13] may be used to predict the probability of occurrence of a peak value of a given distortion metric. This method, which fits the peak experimental data with a model, is used in the current analysis to calculate the expected maximum distortion level for a given operating time within confidence intervals [18].

For S-ducts, the distortion at the Aerodynamic Interface Plane (AIP) is mainly due to the secondary flows and local separation regions within the intake. Recent unsteady CFD studies [11] for similar ducts to those evaluated in this work, highlighted common aspects of the unsteady distortion and identified key coherent structures at the AIP. Previous Stereoscopic Particle Image Velocimetry (PIV) and CFD analysis [9][19] found that the unsteady distortion characteristics of the velocity field were associated with different flow modes. A swirl switching mechanism was identified at the AIP caused by the oscillation of the secondary flows. A vertical 
perturbation mode was also identified due to the shear layer associated with the local separation within the duct. The unsteady distortion at the AIP is commonly quantified by the computation of instantaneous distortion levels based on a 40 probe measurement rake $[4,20,21]$. However these descriptors were initially developed for steady flow and moderate distortion patterns which can inherently filter some localized peak distortion levels at the AIP. There is a need to assess whether these descriptors can be used to not only assess the peak value of the instantaneous distortion but also to characterize the general dynamic of the flow field at the AIP. Delot et al. [22] measured the unsteady total pressure flow field at the exit of an S-duct with a moderate offset $(\mathrm{H} / \mathrm{L}=0.27$, Fig. $1 \mathrm{a})$ for an AIP Mach number $\left(M_{A I P}\right)$ of 0.2 with 40 high bandwidth total pressure probes. Spectral analysis revealed that the unsteady pressure measurements of the two total pressure loss regions on each side of the symmetry plane on the lower half of the AIP were strongly correlated and out of phase. The identification of two main frequencies of $110 \mathrm{~Hz}$ and $220 \mathrm{~Hz}$ led to the hypothesis of associated lateral and longitudinal movements of the streamwise vortices at the AIP. The analysis of the unsteady total pressure field performed by Garnier [23] for a more aggressive S-duct $(\mathrm{H} / \mathrm{L}=0.49$, Fig. 1b) also identified a lateral oscillation at a frequency of 200 $\mathrm{Hz}$ at the AIP for $M_{A I P}=0.2$. Significant levels of energy associated with a frequency range of $250-450 \mathrm{~Hz}$ were also identified near the centre of the AIP. Data from both of these cases is used in the current analysis.

The overall aim of this paper is to assess circumferential and radial distortion statistics and to investigate if EVT can be used to estimate return values and maximum levels of distortion descriptors for complex S-duct flow fields. This paper also provides a systematic assessment of the EVT method based on a de-clustering process and a convergence study to determine if guidelines in terms of acquisition time are sufficient to capture the overall characteristics of extreme distortion events. The methodology developed through this paper is also applied for the first time on unconventional local ring based distortion descriptors which are more appropriate to evaluate local peak distortion events.

\section{Methodology}

\subsection{Experimental facility and test case}

The investigation presented in this paper processes data from two experiments previously performed at ONERA (Modane) using the suck down intake rig R4. The details of the experimental set up can be found in Delot et al. and Garnier [22,23]. For this project, two circular cross-section S-ducts were tested. Both S-duct diffusers have similar design parameters except for the vertical offset $(\mathrm{H} / \mathrm{L})$. For both duct $\mathrm{A}$ and $\mathrm{B}$, the centreline curve is made of two symmetrical arcs of radius $R_{c}$ and maximum angle $\theta_{\text {max }}$ (Table 1 ). The change in the cross section area is defined by Eq.(1):

$$
\frac{D}{D_{\text {in }}}=1+3\left(\frac{D_{A I P}}{D_{\text {in }}}-1\right)\left(\frac{\theta}{\theta_{\max }}\right)-2\left(\frac{D_{A I P}}{D_{\text {in }}}\right)\left(\frac{\theta}{\theta_{\max }}\right)^{3}
$$

For both ducts the inlet diameter was $D_{\text {in }}=133 \mathrm{~mm}$ and the outlet diameter is $D_{\text {out }}=164 \mathrm{~mm}$. The area ratio $A_{\text {in }} / A_{\text {out }}$ was 1.52 for both ducts. For duct A, the offset to diameter ratio $\left(H / D_{i n}\right)$ was 1.34 and the length to diameter ratio $\left(L / D_{\text {in }}\right)$ was 5 . This duct design was experimentally investigated by Wellborn [24] and Zachos et al. [8] as well as in steady [25] and unsteady [11] simulations. Duct B was designed by Garnier [23] with an $H / D_{\text {in }}$ of 2.44 and a slightly shorter length to diameter ratio ( $L / D_{\text {in }}$ ) of 4.95 (Fig. 1, Table 1). Duct B was also evaluated through steady and unsteady CFD simulations [11,19] as well as with S-PIV measurements $[8,9]$. Duct A was designed to achieve a complex three dimensional flow field associated with an unsteady streamwise separation representative of typical diffusing intake configuration. Duct B was designed to achieve a geometry in which the flow distortion would be increased. The idea was to obtain a more representative flow field of future aircraft intake designs where the propulsion system would be deeply integrated to the structure.

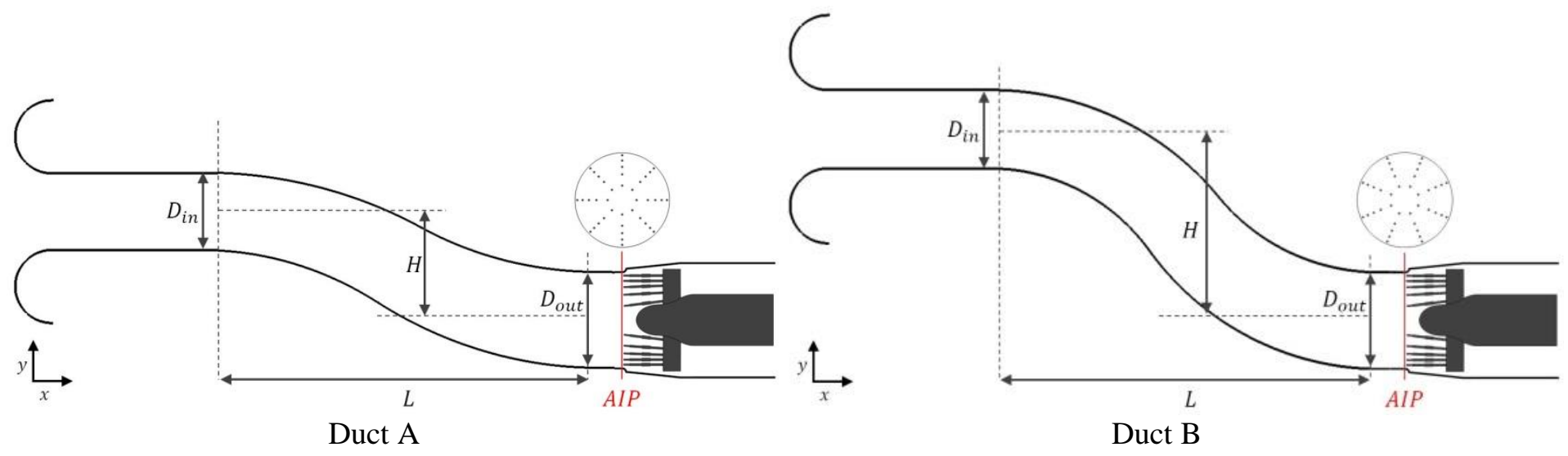

Fig. 1 S-duct configurations and geometry definitions 
Upstream of the S-duct inlet, a constant diameter duct $\left(D_{i n}=133 \mathrm{~mm}\right)$ and a bell mouth section were used for the flow to settle before entering through the intake. Downstream of the S-duct, a constant diameter section $\left(D_{\text {out }}=164 \mathrm{~mm}\right)$ incorporates a 40 Kulite rake located at the AIP. An expansion section with a sudden increase in diameter is placed downstream of the AIP to reduce blockage due to the total pressure rakes (Fig. 1). The flow is then discharged through a constant section duct connected to a sonic throat to regulate the overall mass flow.

Table 1 S-duct design parameters

\begin{tabular}{ccc}
\hline \hline Parameter & Duct A & Duct B \\
\hline $\mathrm{D}_{\text {in }}$ & $133 \mathrm{~mm}$ & $133 \mathrm{~mm}$ \\
$\mathrm{D}_{\text {out }}$ & $164 \mathrm{~mm}$ & $164 \mathrm{~mm}$ \\
$\mathrm{~A}_{\text {out }} / \mathrm{A}_{\text {in }}$ & 1.52 & 1.52 \\
$\mathrm{H} / \mathrm{D}_{\text {in }}$ & 1.34 & 2.44 \\
$\mathrm{~L} / \mathrm{D}_{\text {in }}$ & 5.0 & 4.95 \\
$\theta_{\text {max }} / 2$ & $30^{\circ}$ & $52.55^{\circ}$ \\
$\mathrm{R}_{\mathrm{c}} / \mathrm{D}_{\text {in }}$ & 5 & 3.125 \\
\hline \hline
\end{tabular}

The flow field at the Aerodynamic Interface Plane (AIP) (Fig. 1) was measured with a 40 probe rake using high frequency response pressure transducers with a 5psi differential range (Kulite XCQL093-5D) (Fig. 2b). The rake was designed based on the requirements of the Society of Automotive Engineers (SAE) [4], the rake is made of 8 arms that each include 5 high-bandwidth probes located at radial positions to provide equal area distributions (Fig. 2b). The acquisition frequency of the unsteady total pressure at the AIP was $10 \mathrm{kHz}$ and $20 \mathrm{kHz}$ for duct $\mathrm{A}$ and B, respectively. An additional low-pass Butterworth fourth order filter of $2 \mathrm{kHz}$ was applied on the signal. The total duration of the acquisition was $6 \mathrm{~s}$ for both ducts. For duct $\mathrm{B}$ the rake was rotated by $\theta_{\text {rake }}=22.5$ degrees (Fig. 2b) to offer a more appropriate position of the probes to give relevant information about the loss region at the AIP. The uncertainty associated with the total pressure measurement at the AIP is $10 \mathrm{~Pa}$ which equates to approximately less than $0.1 \%$ of the typical maximum local average total pressure deficit. It represents an uncertainty that ranges from $2 \%$ to $11 \%$ relative to the total pressure fluctuations flow field at the AIP. For duct A the AIP Mach numbers $\left(M_{A I P}\right)$ investigated were 0.2 and 0.36 while for duct B the AIP Mach numbers were 0.2 and 0.4. The Reynolds number range based on the inlet diameter and average velocity the inlet was about 0.5 to $1.5 \times 10^{6}$.

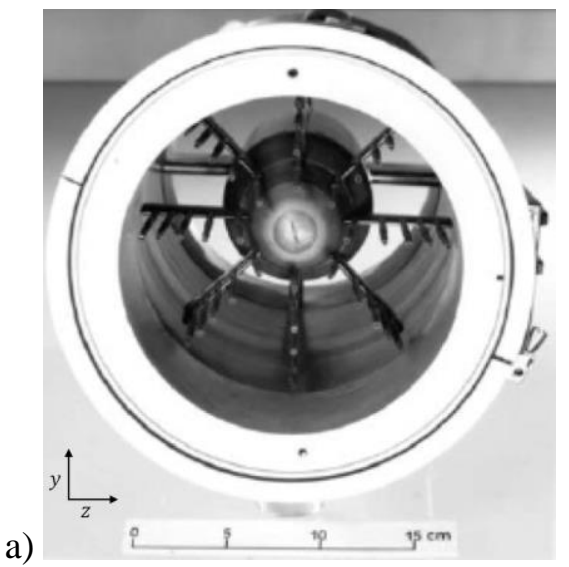

b)

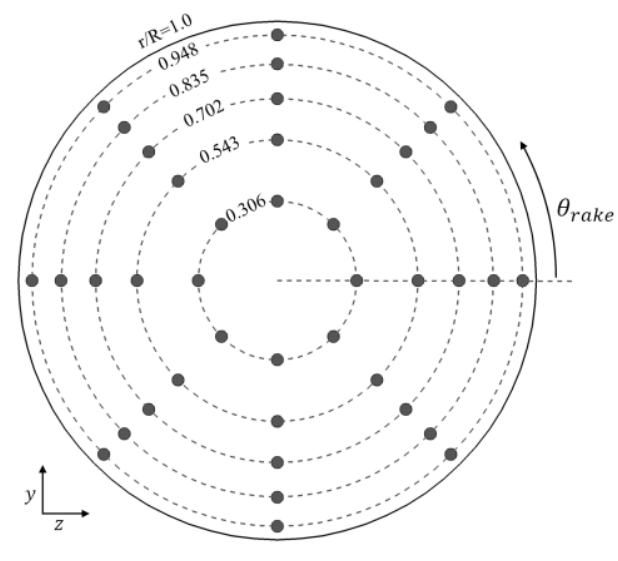

Fig. 2 Photograph of the 40 Kulites rake (a) and representation of the position of the probes at the AIP (b)

\subsection{AIP total pressure distortion assessment}

Distortion descriptors are used to quantify the impact of the change in offset and inlet conditions on the AIP flow field [26]. The distortion is usually divided into two categories of radial or circumferential distortion. The S-ducts efficiencies are assessed through the total Pressure Recovery $P R$ which is the ratio between the area-weighted averaged total pressure at the AIP $\left\langle\bar{P}_{0}{ }_{A I P}\right\rangle$ and the reference pressure $P_{0_{\text {ref }}}$ (Eq.2) as prescribed by the Society of Automotive Engineers (SAE) [4]. In this case the reference total pressure $\left(P_{0_{r e f}}\right)$ is taken as the atmospheric pressure. 


$$
P R=\frac{\left\langle{\overline{P_{0}}}_{A I P}\right\rangle}{P_{0_{\text {ref }}}}
$$

The SAE [4] defined a methodology to assess the total pressure distortion. The descriptors are defined on a radial ring-by-ring basis at the AIP. The circumferential distortion descriptor is defined in terms of extent $(\theta)$, intensity $\left(\Delta P_{C} / P\right)$ and multiple-perrevolution (MPR). These distortion descriptors have been developed in order to provide a way to evaluate inlet flow condition which can be correlated to loss of compressor surge pressure ratio [4].The circumferential pressure descriptors is calculated in terms of low pressure region $\left(P_{0}<\overline{P_{0_{l}}}\right)$ within a given $\mathrm{i}^{\text {th }}$ ring. The extent of a pressure distortion region is defined as the circumferential angle $\theta_{i}^{-}$ over which $P_{0}<\overline{P_{0_{l}}}$. The magnitude of the low pressure region is then calculated as :

$$
\left(\frac{\Delta P_{c}}{P}\right)_{i}=\left(\frac{\overline{P_{0_{\iota}}}-\overline{P_{0_{\text {low }}}}}{\overline{P_{0_{\iota}}}}\right)_{i}
$$

where :

$$
\left(\overline{P_{0_{\text {low }_{l}}}}\right)_{i}=\frac{1}{\theta_{i}^{-}} \int_{\theta_{1}}^{\theta_{2}} P_{0}(\theta)_{i} d \theta
$$

In the case of multiple pressure distortion regions, the circumferential distortion intensity $\left(\frac{\Delta P_{c}}{P}\right)_{i, k}$ and extent $\theta_{i, k}^{-}$are calculated for every low pressure region $k$. The intensity and extent value assigned for the ring $\mathrm{i}$ is the one corresponding to the lowest pressure region with the biggest area which corresponds to the value maximum value of $\left[\left(\frac{\Delta P_{c}}{P}\right)_{i, k} \theta_{i, k}^{-}\right]$. If two lobes of low pressure region are circumferentially separated by a high pressure region with $\theta^{+}$lesser than a defined $\theta_{\text {min }}^{+}$, it is considered as an equivalent one per revolution low pressure region with the extent defined as:

$$
\theta_{i}^{-}=\sum_{k=1}^{Q} \theta_{i k}^{-}
$$

And the intensity as:

$$
\left(\frac{\Delta P_{C}}{P}\right)_{i}=\frac{\sum_{k=1}^{Q}\left(\frac{\Delta P_{C}}{P}\right)_{i k} \theta_{i k}^{-}}{\sum_{k=1}^{Q} \theta_{i k}^{-}}
$$

For this work, $\theta_{\min }^{+}$is taken as $25^{\circ}$ as suggested by the SAE standard [4]. The mean circumferential distortion is calculated as:

$$
\frac{\overline{\Delta P_{C}}}{P}=\frac{1}{5} \sum_{i=1}^{5}\left(\frac{\Delta P_{C}}{P}\right)_{i}
$$

The radial total pressure descriptor is also calculated as the difference between the ring average total pressure $\left(\overline{p 0_{l}}\right)$ and the average total of the AIP $\left(\overline{p 0_{A I P}}\right)$.

$$
\left(\frac{\Delta P_{R}}{P}\right)_{i}=\frac{{\overline{P_{0}}}_{A I P}-\overline{P_{0_{l}}}}{{\overline{P_{0}}}_{A I P}}
$$

For this paper, the radial distortion and circumferential are also defined as follows:

$$
\left(\frac{\Delta P_{R}}{P}\right)_{h u b}=-\left(\frac{\Delta P_{R}}{P}\right)_{1} \quad \text { if } \quad\left(\frac{\Delta P_{R}}{P}\right)_{1}<\left(\frac{\Delta P_{R}}{P}\right)_{5}
$$




$$
\begin{aligned}
& \left(\frac{\Delta P_{R}}{P}\right)_{\text {tip }}=\left(\frac{\Delta P_{R}}{P}\right)_{5} \text { if }\left(\frac{\Delta P_{R}}{P}\right)_{1}>\left(\frac{\Delta P_{R}}{P}\right)_{5} \\
& \left(\frac{\Delta P_{C}}{P}\right)_{\max }=\max \left\{\left(\frac{\Delta P_{C}}{P}\right)_{i}\right\} \text { for } i=1, \ldots, 5
\end{aligned}
$$

\subsection{Extreme Value Theory}

Extreme Value Theory (EVT) is used to model the distribution of extreme events of a given signal. For this application, an extreme event is defined as rare but meaningful instance of the distortion at the AIP likely to adversely impact the hypothetical compression system of an embedded engine. Historically, EVT has been used to assess the peak distortion based on unsteady PR value and distortion descriptors [13] [27]. A distortion screen placed in front of the compressor during a test was generally used to reproduce and amplify the mean perturbed flow field at the exit of S-ducts. This method was recommended by Jacocks and Kneile [27] instead of trying to reproduce a specific engine face pressure pattern. The EVT model estimates the probability of any peak distortion level based on a reduced data set of $n$ independent and identically distributed samples. Therefore, EVT may be used to extrapolate, providing a way of estimating the most probable peak distortion levels which will occur in time periods longer than that of the original data. EVT for inlet distortion was first used by Jacocks [13] to predict the distribution of peak events using the block maxima approach [18]. For this work, the peak over thresholds method is applied to various distortion descriptor signals $X$. It assumes that the limit distribution of the $k$ excesses $\left(Y_{i}\right)$ (Eq.12) for a given threshold $\mu$ behaves as a General Pareto distribution (GPD) (Eq.13). The GPD is a flexible function defined by the shape $\xi$ and scale $\sigma$ parameters. A light-tailed distribution of the GPD has a value of $\xi$ between -0.5 and 0 while the exponential distribution of the GPD has a value of $\xi$ equal to 0 . It is the earlier one that has a particular interest for the application of EVT over distortion signal as when $\xi<0$, the GPD is asymptotic toward the value $\mu-\sigma / \xi$ called the upper bound.

$$
\begin{gathered}
Y_{i}=\left.\left(X_{i}-\mu\right)\right|_{X_{i}>\mu} \\
H(y)=P\{Y \leq y\}=\left\{\begin{array}{l}
1-\left(1+\frac{\xi y}{\sigma}\right)^{-\frac{1}{\xi}} \text { if } \xi \neq 0 \\
1-\exp \left(-\frac{y}{\sigma}\right) \quad \text { if } \xi=0
\end{array}\right. \\
l(\xi, \sigma)=\sum_{i=1}^{k} \log \left(\frac{d H}{d y}\left(Y_{i}, \sigma, \xi\right)\right)=-k \log (\sigma)-(1+1 / \xi) \sum_{i=1}^{k} \log \left(1+\frac{\xi Y_{i}}{\sigma}\right)
\end{gathered}
$$

The main challenge to apply EVT to a given exceedances distribution is the estimation of the model parameters $\xi$, and $\sigma$ for a given threshold $\mu$. The maximum likelihood method (Eq.14) is used to estimate the EVT parameters that maximise the probability of the observed data [18]. From the estimated model parameters, the m-observation return level value $x_{m}$ is obtained with (Eq.15) [18].

$$
x_{m}=\mu+\frac{\sigma}{\xi}\left[\left(m \zeta_{u}\right)^{\xi}-1\right] \text { if } \xi \neq 0
$$

And where $\zeta_{u}$ is the probability of exceeding the threshold equal to $k / n$. The value $x_{m}$ corresponds to the peak value that is exceeded on average once every $m$ observations. The probability $p$ for a given $x_{m}$ is simply the inverse of the number of observation $(p=1 / m)$. In order to estimate the accuracy of the model, the 95\% confidence interval $C I$ (Eq.16) for the return levels $x_{m}$ is derived from the variance of return values assuming that $x_{m}$ follows a normal distribution where $z_{\alpha / 2}=1.96$.

$$
C I=x_{m} \pm z_{\alpha / 2} \sqrt{\operatorname{Var}\left(x_{m}\right)}
$$

The variance of $x_{m}$ is estimated by the delta method (Eq.17) which approximates the error due to each individual parameters of the return function $x_{m}$ based on the gradient $\nabla x_{m}$ (Eq.18) and the variance-covariance matrix VC (Eq.19).

$$
\operatorname{Var}\left(x_{m}\right) \cong \nabla x_{m}^{T} V C \nabla x_{m}
$$




$$
\begin{aligned}
& \nabla x_{m}^{T}=\left[\frac{\partial x_{m}}{\partial \zeta_{u}}, \frac{\partial x_{m}}{\partial \sigma}, \frac{\partial x_{m}}{\partial \xi}\right] \\
& V C=\left[\begin{array}{ccc}
\operatorname{Var}\left(\zeta_{u}\right) & 0 & 0 \\
0 & \operatorname{Var}(\sigma) & \operatorname{Covar}(\xi, \sigma) \\
0 & \operatorname{Covar}(\xi, \sigma) & \operatorname{Var}(\xi)
\end{array}\right]
\end{aligned}
$$

The variance of the parameter $\zeta_{u}$ is estimated from the properties of the binomial distribution (Eq. 20). The variance and covariance for the parameters $\xi$ and $\sigma$ are derived from the maximum likelihood equation (Eq.21-Eq.23).

$$
\begin{gathered}
\operatorname{Var}\left(\zeta_{u}\right)=\frac{\zeta_{u}\left(1-\zeta_{u}\right)}{n} \\
\operatorname{Var}(\xi)=\frac{(1+\xi)^{2}}{k} \\
\operatorname{Var}(\sigma)=\frac{2(1+\xi) \sigma^{2}}{k} \\
\operatorname{Covar}(\xi, \sigma)=-\frac{(1+\xi) \sigma}{k}
\end{gathered}
$$

\section{Results and discussion}

\subsection{Time averaged and unsteady flow field analysis}

The flow field at the Aerodynamic Interface Plane (AIP) is affected by the flow separation within the duct and the classical secondary flows due to the geometry curvature. For the Duct A configuration, Wellborn [24] performed steady velocity measurements at the AIP using a five hole probe and demonstrated the presence a symmetrical pair of vortices. These vortices were also measured using S-PIV by Zachos et al. [8] for the same configuration. A non-uniform total pressure flow field at the AIP is characterized by losses on the lower centre part of the section (Fig. $3 \mathrm{a}-\mathrm{b}$ ). When the S-duct offset is increased from $H / D_{\text {in }}=1.34$ (duct $\mathrm{A}$ ) to 2.44 (duct B) there is a clear impact on the total pressure flow field (Fig. $3 \mathrm{c}-\mathrm{d}$ ). The low pressure region is moved towards the centre of the AIP and has a larger extent. As a result the pressure recovery (Table 2) of the S-duct is reduced from 0.993 for duct A to 0.990 for duct B at $M_{A I P}=0.2$. Furthermore, the adverse pressure gradient established at the second bend for duct $\mathrm{B}$ causes a second loss region at the top of the AIP (Fig. 3c). The increase in AIP Mach number enhances the pressure loss deficit at the AIP for both ducts. This is accompanied by a reduction in pressure recovery from 0.993 to 0.971 for duct A and from 0.990 to 0.953 for duct B (Table 2). It is also illustrated by the greater loss from about $0.5 q_{A I P}$ to $0.9 q_{A I P}$ in the low pressure region for both configurations (Fig. $3 \mathrm{~b}$ and d).

Several previous studies demonstrated that the fluctuating aspect of the distortion can severely alter the dynamic response of the compression system and the onset of undesirable events [6]. The fluctuations in total pressure at the AIP are related to the unsteadiness of the secondary flows [9] as well as the centreline separation [11]. The use of proper orthogonal decomposition (POD) applied to the S-PIV measured velocity data [9] and unsteady CFD simulations [19] revealed that the velocity flow field unsteadiness was dominated by the swirl switching mechanism [28]. Relative to the dynamic pressure, the total pressure fluctuations are moderately affected by Mach number for both duct configurations. For duct A $\left(H / D_{i n}=1.34\right)$, the maximum level of fluctuations varies from $\sigma_{\left(P_{0}\right)} / q_{A I P}=$ 0.3 to 0.37 for the low and high Mach number respectively (Fig. $4 \mathrm{a}$ and b). The maximum total pressure fluctuations $\left(\sigma\left(P_{0}\right) / q_{A I P}\right)$ for duct B increases from 0.36 to 0.40 for an AIP Mach number $\left(M_{A I P}\right)$ of 0.2 and 0.4 respectively (Fig. $4 \mathrm{c}$ and d). For both duct A and B, the periphery of the main loss in total pressure region (Fig. 3) is associated with high values of total pressure fluctuations (Fig. 4). However, the change in S-duct offset from $H / D_{i n}=1.34$ to 2.44 significantly affects the average total pressure unsteadiness at the AIP where $\left\langle\sigma_{P_{0}}\right\rangle /\left\langle q_{A I P}\right\rangle$ increases from 0.183 to 0.216 at $M_{A I P}=0.2$ (Table 2) for duct A and B respectively. This is explained by the very low level of total pressure unsteadiness on the upper half of the AIP for duct A with values of $\sigma_{\left(P_{0}\right)} / q_{A I P}$ close to 0 . Therefore the stronger secondary flows due to the offset increase not only promote the losses in total pressure but also increase the overall unsteadiness of the flow field at the AIP. 


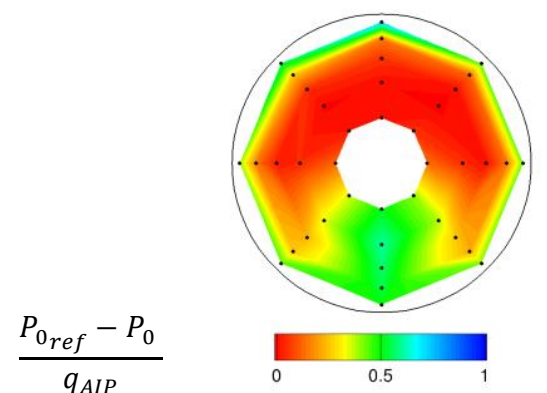

a) Duct $\mathrm{A} M_{A I P}=0.2$

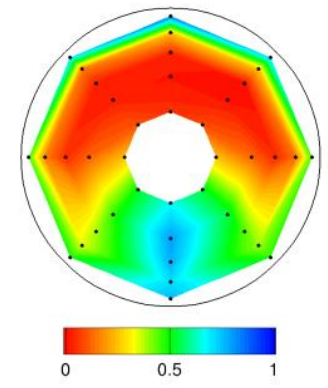

b) Duct A $M_{A I P}=0.36$

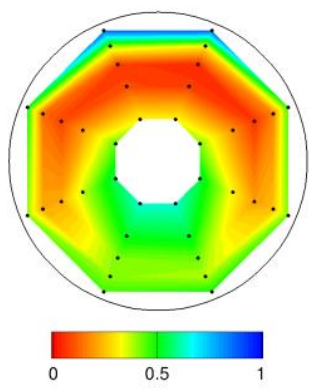

c) Duct B $M_{A I P}=0.2$

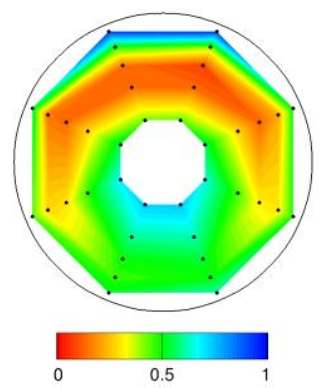

d) Duct B $M_{A I P}=0.4$

Fig. 3 Distribution of the non-dimensionalized time averaged total pressure loss flow field at the AIP

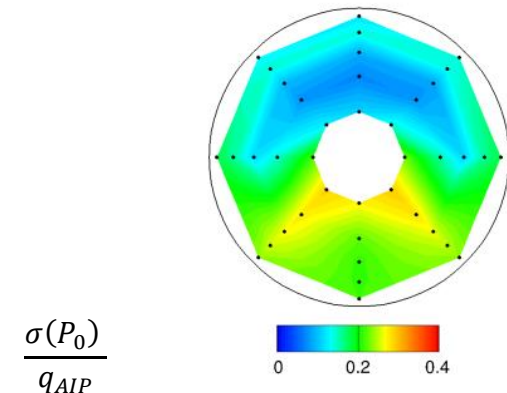

a) Duct $\mathrm{A} M_{A I P}=0.2$

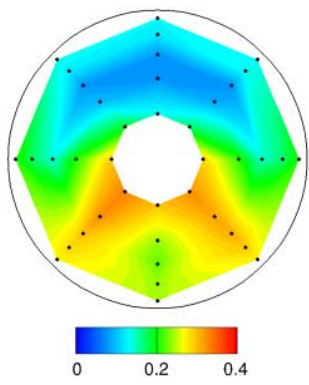

b) Duct A $M_{A I P}=0.36$

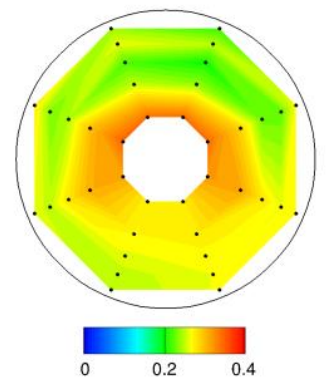

c) Duct B $M_{A I P}=0.2$

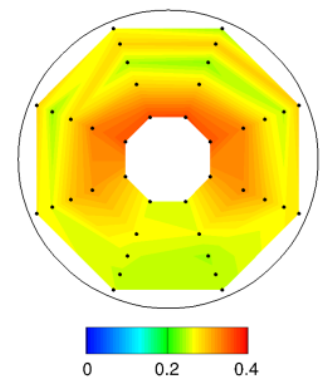

d) Duct B $M_{A I P}=0.4$

Fig. 4 Distribution of the non-dimensionalized total pressure fluctuations at the AIP

Table 2 Weighted averaged statistics

\begin{tabular}{ccccc}
\hline \hline & \multicolumn{2}{c}{ Duct A } & \multicolumn{2}{c}{ Duct B } \\
\hline$M_{A I P}$ & 0.2 & 0.36 & 0.2 & 0.4 \\
$\overline{\left\langle P_{0}\right\rangle} / P_{0_{i}}$ & 0.993 & 0.971 & 0.990 & 0.953 \\
$\overline{s t d\left(P_{0}\right)} /\left\langle q_{A I P}\right\rangle$ & 0.183 & 0.217 & 0.276 & 0.304 \\
\hline \hline
\end{tabular}

\subsection{Unsteady distortion assessment}

Several descriptors have been developed in order to analyse the flow distortion at an AIP. These descriptors attempt to provide a characterisation of the non-uniform flow field at the AIP in terms of intensity and general shape. The SAE pressure based descriptors have been developed in order to provide a way to evaluate inlet flow condition which can be correlated to loss of compressor surge pressure ratio [4].

Table 3 Summary of distortion statistics

\begin{tabular}{cccccc}
\hline \hline & \multicolumn{2}{c}{ Duct A } & \multicolumn{2}{c}{ Duct B } \\
\cline { 2 - 6 } & $M_{\text {AIP }}$ & 0.2 & 0.36 & 0.2 & 0.4 \\
\hline \multirow{\Delta}{*}{$P_{C} / P$} & $<>$ & 0.006 & 0.025 & 0.007 & 0.028 \\
& $\sigma$ & 0.002 & 0.006 & 0.002 & 0.006 \\
& $\max$ & 0.013 & 0.048 & 0.014 & 0.054 \\
\hline \multirow{3}{*}{$\left(\Delta P_{C} / P\right)_{\max }$} & $<>$ & 0.009 & 0.035 & 0.010 & 0.038 \\
& $\sigma$ & 0.002 & 0.008 & 0.002 & 0.008 \\
& $\max$ & 0.019 & 0.069 & 0.023 & 0.089 \\
\hline \multirow{3}{*}{$\left(\Delta P_{R} / P\right)_{\text {hub }}$} & $<>$ & -0.007 & -0.025 & -0.009 & -0.037 \\
& $\sigma$ & 0.003 & 0.011 & 0.004 & 0.016 \\
& $\min$ & -0.022 & -0.076 & -0.025 & -0.097 \\
\hline \multirow{3}{*}{$\left(\Delta P_{R} / P\right)_{\text {tip }}$} & $<>$ & 0.007 & 0.026 & 0.007 & 0.028 \\
& $\sigma$ & 0.002 & 0.007 & 0.002 & 0.010 \\
& $\max$ & 0.014 & 0.049 & 0.018 & 0.067 \\
\hline \hline
\end{tabular}



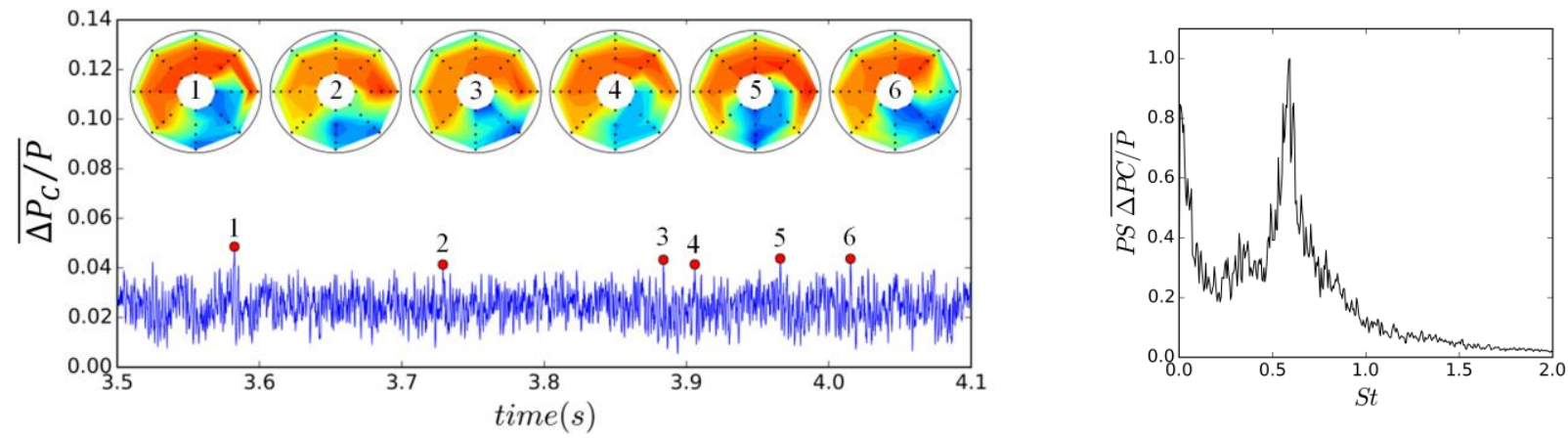

Fig. $5 \overline{\Delta P_{C} / P}$ time signal and power spectrum for duct $\mathrm{A}$ at $M_{A I P}=0.36$
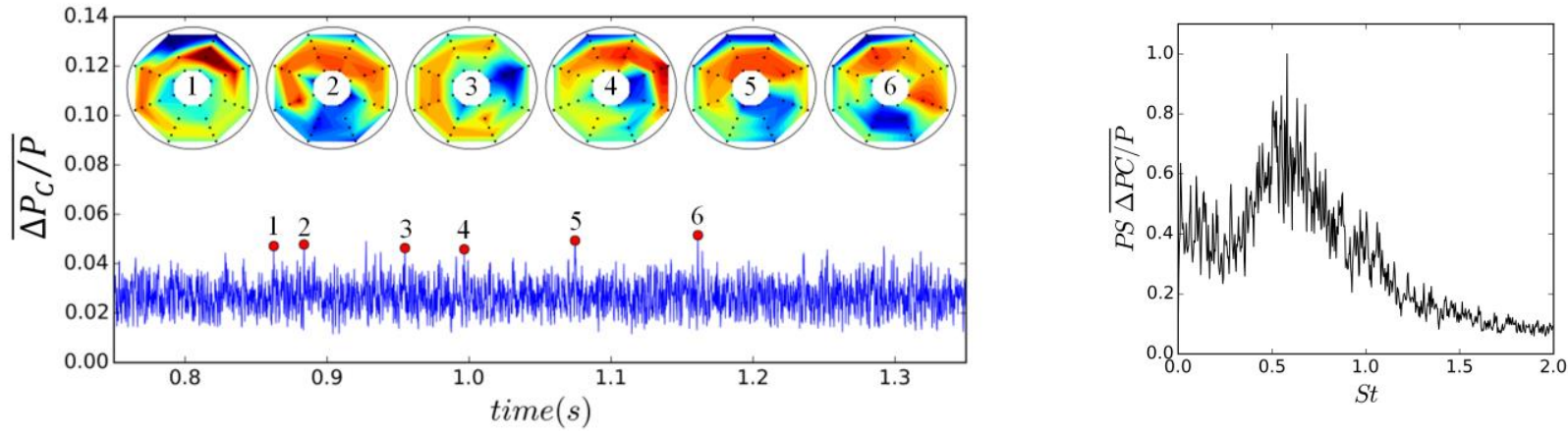

Fig. $6 \overline{\Delta P_{C} / P}$ signal and power spectrum for duct $B$ at $M_{A I P}=0.4$
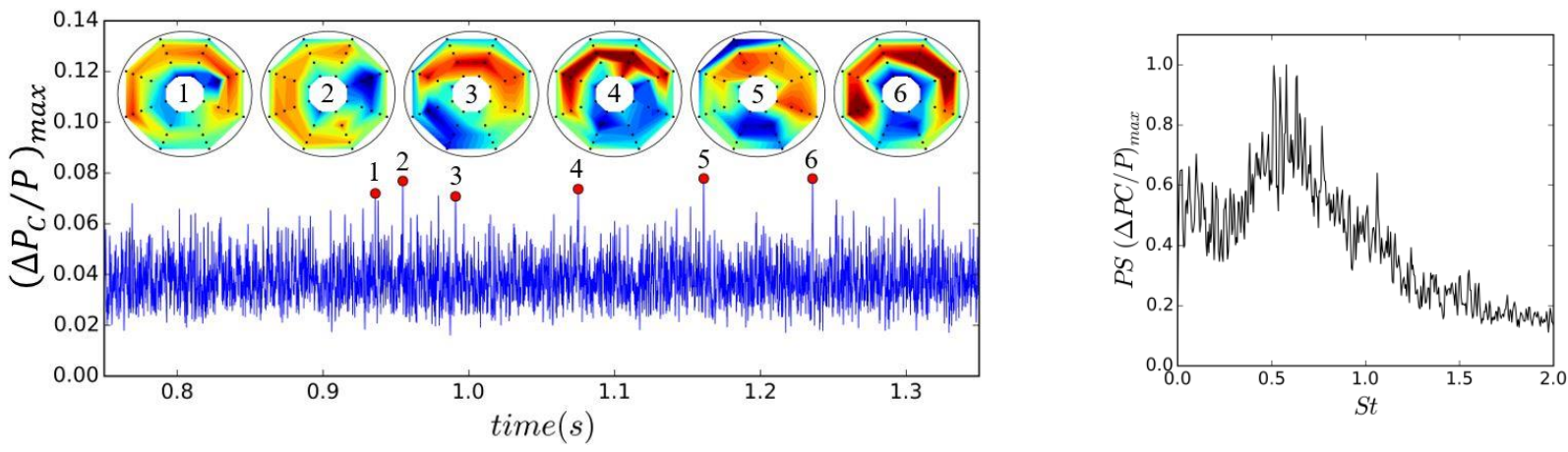

Fig. $7\left(\Delta P_{C} / P\right)_{\max }$ signal and power spectrum for duct $B$ at $M_{A I P}=0.4$
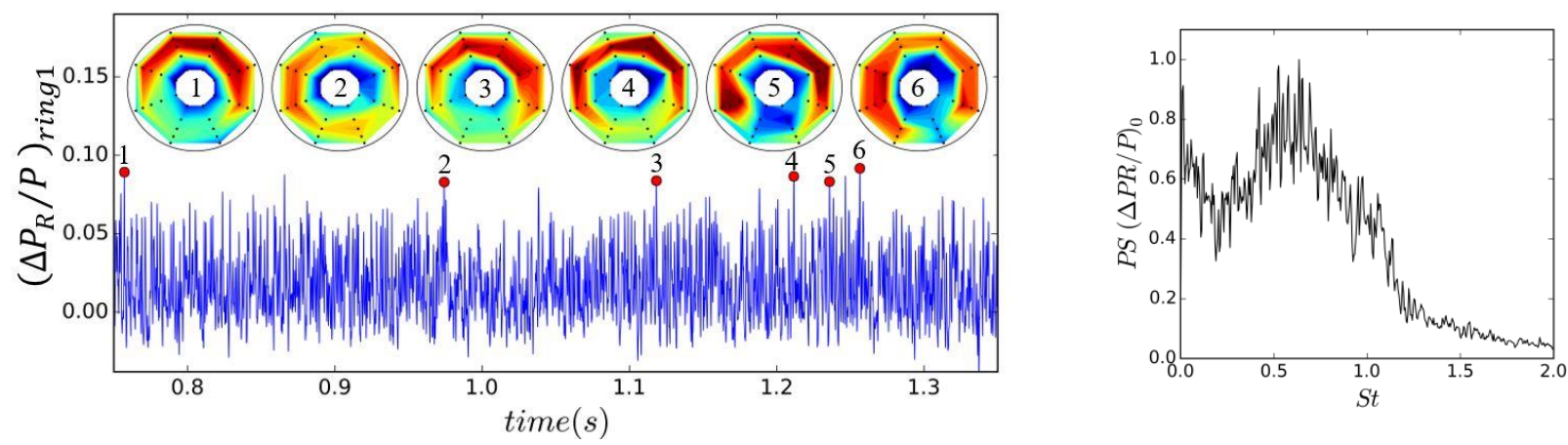

Fig. $8\left(\Delta P_{R} / P\right)_{\text {ring } 1}$ signal and power spectrum for duct B at $M_{A I P}=0.4$

Although both ducts have similar distortion mechanisms as identified through the Proper Orthogonal Decomposition (POD) analysis performed on the $3 \mathrm{D}$ velocity flow field [9], the change in offset from $H / D_{\text {in }}=1.34$ (duct $\mathrm{A}$ ) to 2.34 (duct $\mathrm{B}$ ) induces stronger secondary and separated flows within the duct [11]. However, the statistics of the mean circumferential descriptor $\overline{\Delta P_{C} / P}$ for both duct $\mathrm{A}$ and $\mathrm{B}$ at similar operating conditions $\left(M_{A I P}=0.2\right)$ does not indicate any major effect of the duct offset. Therefore the 
clear increase of flow unsteadiness (Fig. 4) due to the change in offset from $H / D_{\text {in }}=1.34$ to 2.44 for duct A and B respectively does not affect the circumferential distortion for $M_{A I P}=0.2$ (Table 3). This demonstrates the limitation of the use of SAE time averaged total pressure based descriptors to compare the aerodynamic characteristics of two different S-duct geometries. The surge of an engine or the onset of rotating stall cells within the compressor is generally due to instantaneous peak distortion levels that were not considered within the design stage [15]. The assessment of peak distortion associated with large deviation from the time averaged total pressure field has to be taken into account. The instantaneous snapshots identified for a given peak value of $\overline{\Delta P_{C} / P}$ demonstrates the wide variety of flow topologies at the AIP for duct A and B (Fig. 5, Fig. 6). The large total pressure losses at the AIP are not only centred around the time averaged main loss region but also at the upper periphery of the section. A peak value in mean circumferential distortion $\overline{\Delta P_{C} / P}$ is associated with a flow pattern with significant losses in total pressure. However, as $\overline{\Delta P_{C} / P}$ is calculated as the averaged value of the circumferential distortion obtained for each ring, high distortion events which are more localised to the region of a smaller number of rings are less evident in this metric. Therefore, the maximum circumferential distortion value $\left(\Delta P_{C} / P\right)_{\text {max }}$ obtained between the 5 rings is also used to assess the peak distortion events (Fig. 7). The mean and maximum value of $\left(\Delta P_{C} / P\right)_{\max }($ Table 3$)$ for duct $\mathrm{A}$ are about $30 \%$ greater than $\overline{\Delta P_{C} / P}$ for duct $\mathrm{A}$ at both $M_{A I P}$ conditions. This is also the case for duct B. Thus the assessment for the mean circumferential descriptors $\overline{\Delta P_{C} / P}$ statistics is on average $30 \%$ lower than $\left(\Delta P_{C} / P\right)_{\text {max }}$. It is therefore important to assess the total pressure distortion on a ring based analysis considering that strong local distortion events can also trigger adverse events for the compressor [29]. The use of the radial distortion descriptor $\Delta P_{R} / P$ can assess whether a distortion event is dominant at the hub $\left(\Delta P_{R} / P<0\right)$ or at the tip $\left(\Delta P_{R} / P>0\right)$ (section 2.2). For duct A, similar levels of time-averaged radial distortion are noted for the hub and tip. However, the maximum absolute value of radial distortion $\max \left(\left|\left(\Delta P_{R} / P\right)\right|\right)$ is higher at the hub than at the tip with 0.022 and 0.014 respectively at $M_{\text {in }}=0.2$. The change in $\mathrm{S}$-duct offset from $\mathrm{H} / D_{\text {in }}=1.34$ to 2.44 increases the absolute time averaged hub distortion $\left\langle\left(\Delta P_{R} / P\right)_{h u b}\right\rangle$ from 0.007 to 0.009 and $\max \left(\left|\left(\Delta P_{R} / P\right)_{\text {hub }}\right|\right)$ from 0.022 to 0.025 at $M_{\text {in }}=0.2$. This is explained by the presence of events where the loss in total pressure region has migrated toward the centre of the AIP which considerably reduce the inner ring total pressure relative to $\overline{P_{0 A I P}}$ for duct $\mathrm{B}$ (Fig. 8). The time averaged tip distortion is not particularly affected by the change in offset. However, the maximum value varies from 0.014 to 0.018 for duct A and B respectively at $M_{\text {in }}=0.2$. Therefore the radial distortion metrics for duct B characterises an environment which may be more detrimental for a compressor rotor blade with the tendency of the low pressure region to migrate toward the centre of the AIP.

Joint-probability cloud maps were first used in a duct flow analysis context by Gil Prieto et al. [9] to evaluate the relationship between two different swirl based descriptors as well as the probability that an event arises. In this work, it is applied for the first time to the measured radial and maximum circumferential total pressure descriptors calculated for duct A and B (Fig. 9). The axis of the plots are discretized with 100 segments which provide a resolution of 0.002 and 0.001 for $\Delta P_{R} / P$ and $\left(\Delta P_{C} / P\right)_{\text {max }}$ respectively. For duct $\mathrm{A}$, about $91 \%$ of the events are associated with tip radial distortion $\left(\Delta P_{R} / P>0\right)$ which is explained by the main loss region being most of the time located near the lower periphery of the AIP. However, the range of the radial distortion descriptors for the hub dominated events is larger with $\max \left(\left|\Delta P_{R} / P\right|\right)$ equal to 0.076 compare with 0.049 for tip dominated events. Thus although hub radial events are less common for duct A, they are promoted by a wider range of peak values which can also be associated with large circumferential distortion events (Fig. 9a).

For duct $\mathrm{B}$, the change in flow topology due to the increase in offset from $H / D_{\text {in }}=1.33$ to 2.44 is characterized by the loss in total pressure located at the centre of the AIP (Fig. 3). As a result, the proportion of hub and tip events relative to $\Delta P_{R} / P=0$ is more symmetric (Fig. 9b). However a more scattered distribution of the points with an increase in range arises from the increased unsteadiness of the flow field (Fig. 9b) which demonstrated large deviation from the time averaged distortion descriptors. The peak values of the circumferential distortion $\left(\left(\Delta P_{C} / P\right)_{\max }>0.12\right)$ are associated with a broadband range of $\Delta P_{R} / P$ from -0.05 to -0.8 for hub distortion events. The joint probability map does not indicate any clear relationship between radial and circumferential distortion events. For both ducts, peak value of circumferential distortion can be associated with a large range of radial distortion that can be either hub or tip dominated. These results demonstrate that circumferential and radial total pressure distortion metrics are not dominated by a clear flow mechanism as it is the case for the swirl distortion [9]. The joint probability map also clearly highlights the presence of peak hub radial distortion associated with large circumferential distortion. This is especially true when the main perturbation is located at the hub of the AIP (Fig. 8 event 5).

The evaluation of the total pressure flow field at the AIP demonstrated similar level of circumferential distortion for both ducts although a clear increase of the unsteadiness is noted for duct B. The spatially averaged distortion descriptors can mask significant local peak circumferential distortion events which may adversely affect the engine response. As future intake designs tend towards more aggressive S-duct geometries which promote local flow distortion, the use of ring based distortion descriptors to evaluate instantaneous peak value could be a more appropriate method to evaluate extreme events. 


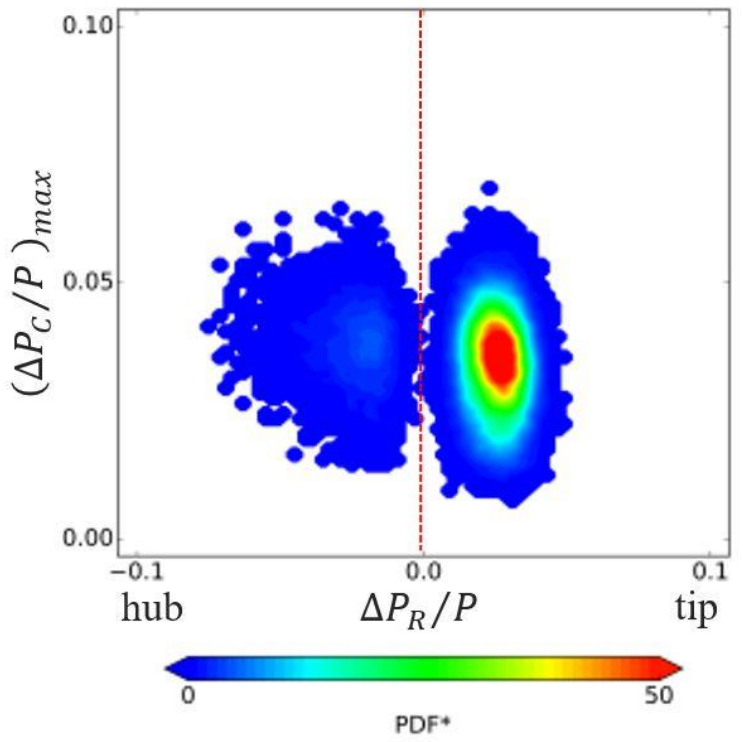

Duct A $M_{A I P}=0.36$

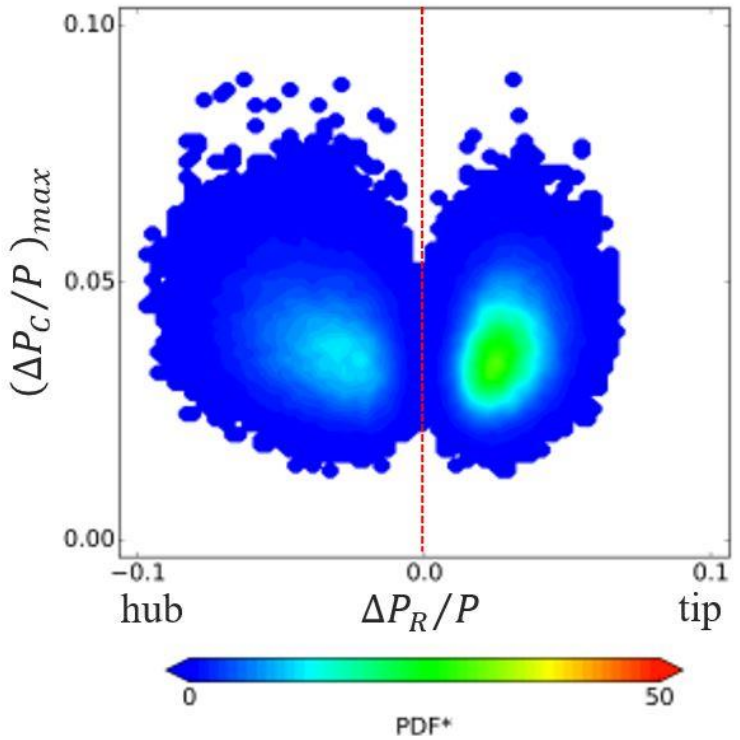

Duct B $M_{A I P}=0.4$

Fig. 9 Joint Probability graphs for $\left(\Delta P_{R} / P\right)-\left(\Delta P_{C} / P\right)_{\max }$

\subsection{Extreme value theory}

The identification of peak distortion events which can adversely affect the compression system highlights the limits of timeaveraged and RMS approaches. Extreme Value Theory (EVT) is a more sophisticated technique which predicts the extreme level of distortion that could occur based on a reduced data set. EVT is applied on the distortion descriptor signal presented in section 2.3 for duct $\mathrm{A}$ and $\mathrm{B}$ at $M_{A I P}$ of 0.36 and 0.4 respectively.

For duct A, the distortion descriptors are obtained from the AIP total pressure field acquired at a frequency of $10 \mathrm{kHz}$ with a low pass filter of $2 \mathrm{kHz}$. The acquisition frequency for duct B is $20 \mathrm{kHz}$ and filtered at $2 \mathrm{kHz}$. The sample size for duct A and B are 60,000 and 119,860, respectively, which provides an acquisition time of around $6 \mathrm{~s}$ for both ducts. Although both ducts share similar levels of mean circumferential distortion $\left(\overline{\Delta P_{C} / P}\right)$ (Table 3), some major differences can be noted within the flow pattern for peak distortion events. Duct A has a relatively distortion free upper half of the duct (Fig. 4). As a result, peak distortion events can often be characterised by amplification of the time average total pressure losses (Fig. 5). This type of flow is classified according to Jacocks and Kneile [27] as a medium turbulent field where peak distortion events are broadly similar to the time averaged flow field. Duct B can be classified as a highly turbulent flow field where the instant total pressure patterns of peak distortion events are dissimilar from the steady state pattern (Fig. 6). The application of EVT on this type of flow is less common and not previously reported in the open literature.

For both ducts, the spectral content of the distortion descriptors has a maximum frequency around $\mathrm{St}=1.1(f \cong 900 \mathrm{~Hz})($ Fig. 5 , Fig. 6) which is less than half of the $2 \mathrm{kHz}$ low pass filter used during the experiment. As a result, the statistical characteristics of the distortion metric may not be independent and a single peak of distortion level could be captured by more than one temporal measurement. This characteristic is called peak clustering [13] and violates the typical formal requirement for EVT analysis that it should be applied to a statistically independent variable [18]. This assumption generally makes temporally resolved signals not appropriate candidates for EVT and no standard techniques exist to address peak clustering [18]. One approach is to pre-process the temporal signal to de-cluster the data. The main purpose is to filter the signal to isolate independent peak values that will fit the General Pareto Distribution (GDP). For this research, the signal is divided into a set blocks for which the local maxima of the signal is computed. A block size which is too small will lead to dependent block maxima while an excessively large block size will reduce the number of exceedances and possibly discard peak that could have been considered as independent $[1,30]$. Therefore a systematic assessment is performed in order to investigate the effect of the de-clustering frequency and to determine the robustness of the EVT for the prediction for return values.

The EVT method used in this research is the threshold model for which a threshold $\mu$ needs to be selected [18]. The threshold is employed to identify the exceedances used to fit the model. Analysis of the different EVT parameters obtained from a systematic assessment of the various level thresholds is performed to select the optimum $\mu$. Above a valid threshold, the model shape and scale parameters $\left(\xi, \sigma^{*}=\sigma-\xi U\right)$ (section 2.3) should remain constant after allowance for confidences intervals. The mean excess should also linearly vary with the threshold increase [18] (Fig. 10a). However a wide range of thresholds can satisfy these requirements. For this study, the selected threshold also minimises the root mean squared (RMS) error between the predicted and observed quantiles (Fig. 10b) as recommended by Coles [18]. 


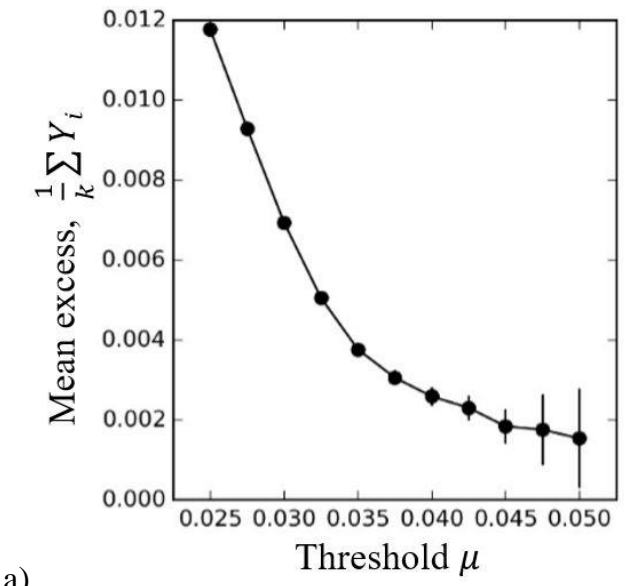

a)

Fig. 10 Example of mean excess (a) and RMS quantiles error (b) for different thresholds based on the $\overline{\Delta P_{C} / P}$ signal for duct $B$

\subsubsection{EVT de-clustering assessment}

The effect of the frequency used to de-cluster the distortion signal over the return plots has been investigated for the Strouhal numbers $\left(S t_{d}\right)$ of $0.25,0.5,0.75$ and 1.0. These frequencies correspond to the range that contains most of the energy associated with the time dependent signal of the circumferential distortion descriptors $\overline{\Delta P_{C} / P}$ (Fig. 5, Fig. 6). The aim is to de-cluster the signal into multiple blocks in order to process only the clusters' maxima which are assumed to be independent. Several thresholds have been tested for the range of de-clustered signals. The selected thresholds are $\mu=0.035$ and 0.037 for duct $\mathrm{A}$ and $\mathrm{B}$, respectively which minimise the root mean square (RMS) error between the predicted and actual excess value. For duct A, the signal is de-clustered into 1131 blocks for the range of frequency $\left[S t_{d}=0.25-1.0\right]$. As a result, the length of the signal is reduced when $S t_{d}$ is increased and the number of exceedances $k$ decreases from 658 to 250 for $S t_{d}=0.25$ and $\mathrm{St}=1.0$ respectively (Table 4 ). For duct B, the signal is de-clustered into 1228 blocks with a number of exceedance varying from 827 to 337 for $S t_{d}=0.25$ and 1.0 respectively. The EVT is able to associate a model that reproduces the experimental peak value for the circumferential descriptor $\overline{\Delta P_{C} / P}$ (Fig. 11, Fig. 12). The return period corresponds to the time of acquisition for which the associated peak level is expected to be exceeded once on average. The return period is derived from the probability $p$ and the acquisition frequency used to de-cluster the signal.

The quality of the EVT model is checked with the comparison of the probability and quantiles distribution of the measured and modelled data (Fig. 13). The return period is generally used to predict the maximum peak value probability for an operating time longer than the available test data. The extrapolation uncertainty is typically based on the $95 \%(C I)$ confidence intervals obtained from the variance error for the return levels $x_{m}$ (section 2.3). For any signal, the estimated confidence intervals increase with return period due to the greater uncertainty associated with the prediction of rare events (Fig. 11 \& Fig. 12). It is common practice to not extrapolate peak values more than 100 times $\left(P_{100 t}\right)$ the available time of observation $t[13]$.

Table 4 Sensitivity of the de-clustering frequency with a constant number of blocks

\begin{tabular}{cccccccccc}
\hline \hline Case & $S t_{d}$ & $\mu$ & $N_{b}$ & $N_{s}$ & $k$ & $U_{b}$ & $C I_{U_{b}}$ & $P_{600 s}$ & $C I_{P_{600 s}}$ \\
\hline & 0.25 & & 1131 & 59978 & 658 & 0.050 & \pm 0.0028 & 0.049 & \pm 0.0019 \\
& 0.5 & & 1131 & 29989 & 395 & 0.048 & \pm 0.0033 & 0.047 & \pm 0.0023 \\
Duct A & 0.75 & & 1131 & 19993 & 281 & 0.050 & \pm 0.0056 & 0.048 & \pm 0.0034 \\
& 1.0 & & 1131 & 14994 & 250 & 0.161 & \pm 0.0781 & 0.060 & \pm 0.0142 \\
\hline & 0.25 & & 1228 & 119876 & 827 & 0.055 & \pm 0.0029 & 0.056 & \pm 0.0020 \\
& 0.5 & & 1228 & 59938 & 544 & 0.059 & \pm 0.0039 & 0.056 & \pm 0.0039 \\
Duct B & 0.75 & & 1228 & 39959 & 403 & 0.067 & \pm 0.0160 & 0.060 & \pm 0.0070 \\
& 1.0 & & 1228 & 29969 & 337 & 0.071 & \pm 0.0199 & 0.061 & \pm 0.0085 \\
\hline \hline
\end{tabular}




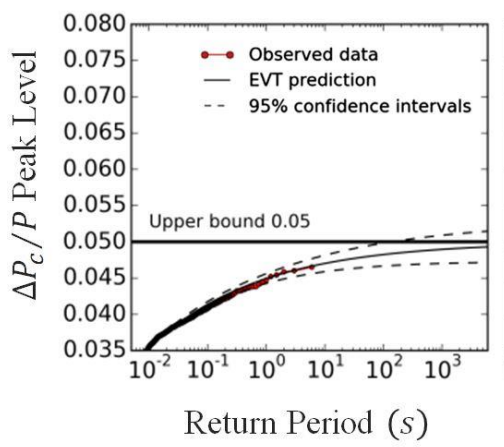

a) $S t_{d}=0.25$

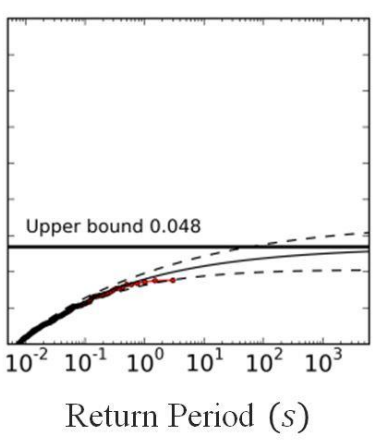

b) $S t_{d}=0.5$

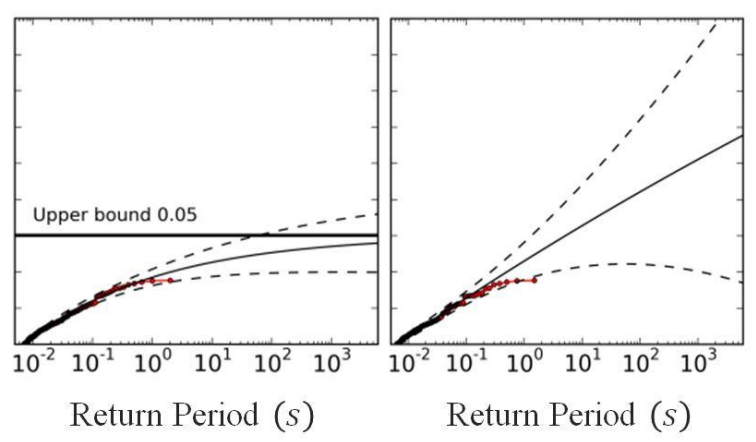

c) $S t_{d}=0.75$

d) $S t_{d}=1.0$

Fig. 11 Distribution of the return plots for a range of de-clustering frequencies with a constant number of blocks for duct $\mathrm{A}$

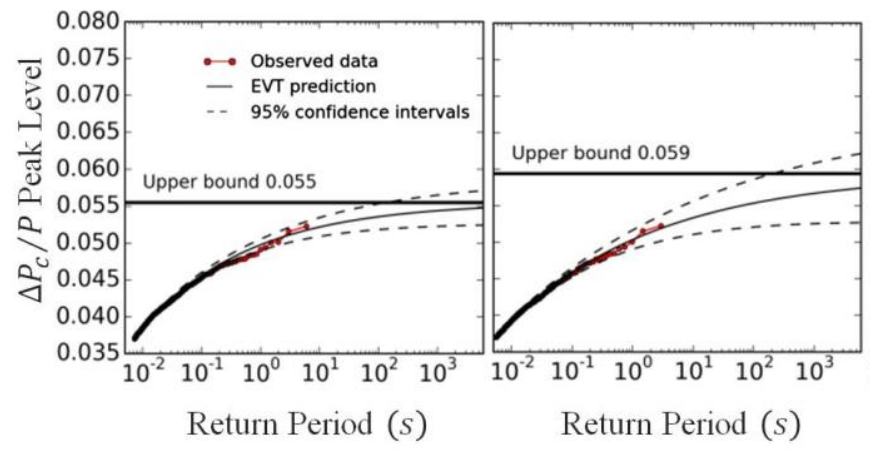

a) $S t_{d}=0.25$

b) $S t_{d}=0.5$

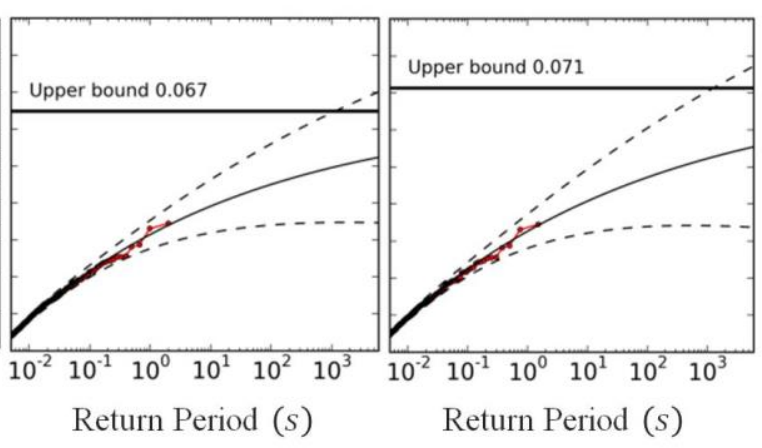

c) $S t_{d}=0.75$

d) $S t_{d}=1.0$

Fig. 12 Distribution of the return plots for a range of de-clustering frequencies with a constant number of blocks for duct $B$

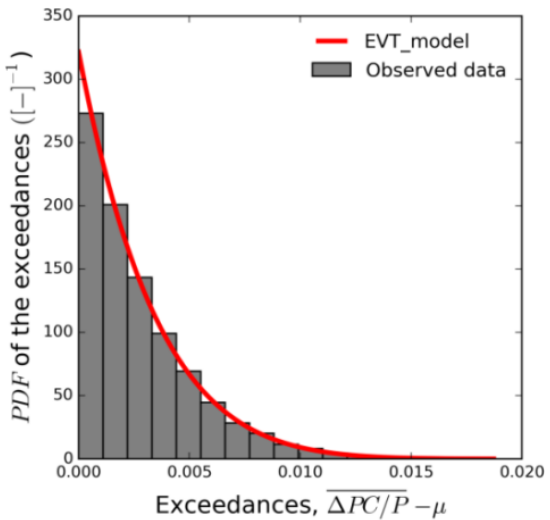

a) b)

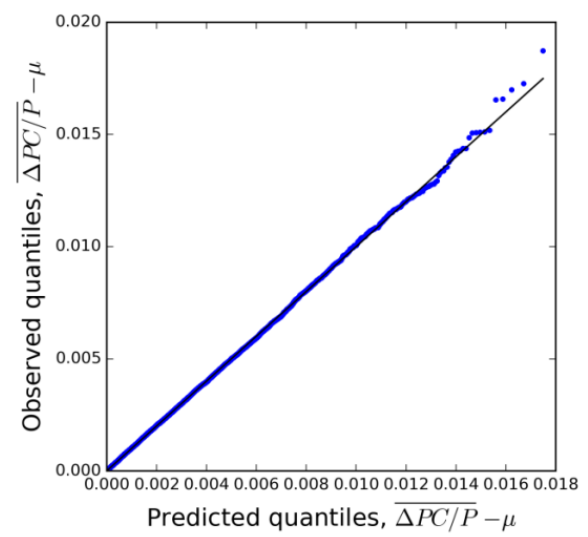

Fig. 13 Comparison between observed data and the EVT model predictions for $\overline{\Delta P_{C} / P}$ for duct B

The sensitivity of the EVT model to $S t_{d}$ is evaluated with the return value for a 600 s period $\left(P_{600 s}\right)$ and the upper bound $\left(U_{b}\right)$. The upper bound is the estimation of the peak distortion value achieved at an infinite return period derived from the general Pareto distribution's asymptote. For duct A, although the number of exceedances $k$ is reduced from 658 to 281 for an increase $S t_{d}$ from 0.25 to 0.75 , the change of $U_{b}$ and $P_{600 s}$ remain negligible (Table 4). However, there is a clear impact on the confidence interval which is doubled for the upper bound $\left(C I_{U_{b}}\right)$ and increased by $80 \%$ for $P_{600 s}\left(C I_{P_{600 s}}\right)$ (Table 4$)$. For the de-clustering frequency of $S t_{d}=1.0$, the EVT model estimates a return plot (Fig. 11, $S t_{d}=1.0$ ) with a very high upper bound of $0.161 \pm 0.078$. The return value for $600 \mathrm{~s}$ is also increased with unacceptable confidence intervals of $C I_{P_{600 s}}=0.014$. For duct $\mathrm{B}$, the upper bound estimation increase from 0.055 to 0.071 as $S t_{d}$ varies from 0.25 to 1.0 . As for duct A, the confidence intervals $\sigma_{U_{b}}$ and $\sigma_{P_{600 s}}$ monotonically increase with the change in $S t_{d}$.

The effect of $S t_{d}$ on the EVT return plot is also investigated for the full signal. The distortion descriptor $\overline{\Delta P_{C} / P}$ signal is declustered with the same $S t_{d}$ range as used previously $\left(S t_{d}=[0.25-1.0]\right)$. The block number is no longer constant and varies from 1131 to 4525 for duct A and from 1228 to 4915 for duct B as $S t_{d}$ increase from 0.25 to 1.0 (Table 5). For duct A, $P_{600 s}$ is not affected with a constant estimation of $\overline{\Delta P_{C} / P}=0.049$ for every $S t_{d}$ investigated. The estimation of $U_{b}$ is slightly increased from 0.050 to 
0.052 when $S t_{d}$ varies from 0.25 to 1.0 (Table 5). The variation of the confidence intervals is also very small and demonstrates that overall for duct $\mathrm{A}$ the frequency at which the signal is de-clustered does not affect the return levels as long as the number of exceedances is sufficient. For duct B, the increase in $S t_{d}$ from 0.25 to 1.0 increases the confidence intervals for $C I_{P_{6005}}$ from 0.0020 to 0.0028 with a negligible variation of $P_{600 s}$. The upper bound estimation $\left(U_{b}\right)$ also varies from 0.055 to 0.060 when the de-clustering frequency is increased from 0.25 to 1.0 with a concomitant increase of the $95 \%$ upper bound confidence intervals $\left(C I_{U_{b}}\right)$ from 0.029 to 0.058 . Therefore for the range of $S t_{d}$ investigated, the de-clustering of the data tends to affect only the confidence intervals for a maximum expected extrapolation of 100 times the acquisition period which for this case is a projected 600s period. A similar analysis was performed for a constant number of exceedances $k$. The signal was de-clustered with $S t_{d}$ ranging from 0.25 to 1.0. However the number of samples used was adjusted in order to maintain a high level of about 800 exceedances. The return plots and upper bound estimation were not significantly changed which showed a low sensitivity relative to the de-clustering frequency.

Table 5 Sensitivity of the de-clustering frequency $\left(S t_{d}\right)$

\begin{tabular}{cccccccccc}
\hline \hline Case & $S t_{d}$ & $\mu$ & $N_{b}$ & $N_{s}$ & $k$ & $U_{b}$ & $C I_{U_{b}}$ & $P_{600 s}$ & $C I_{P_{600 s}}$ \\
\hline & 0.25 & & 1131 & & 658 & 0.050 & \pm 0.0028 & 0.049 & \pm 0.0019 \\
& 0.5 & & 2262 & & 816 & 0.051 & \pm 0.0037 & 0.049 & \pm 0.0021 \\
Duct A & 0.035 & 3394 & 59978 & 890 & 0.052 & \pm 0.0038 & 0.049 & \pm 0.0022 \\
& 0.75 & & & & & \\
& 1.0 & & 4525 & & 931 & 0.052 & \pm 0.0038 & 0.049 & \pm 0.0022 \\
\hline & 0.25 & & 1228 & & 827 & 0.055 & \pm 0.0029 & 0.056 & \pm 0.0020 \\
& 0.5 & & 2457 & & 1084 & 0.057 & \pm 0.0038 & 0.055 & \pm 0.0024 \\
Duct B & 0.75 & 0.037 & 3686 & 119876 & 1227 & 0.059 & \pm 0.0046 & 0.055 & \pm 0.0027 \\
& 1.0 & & 4915 & & 1295 & 0.060 & \pm 0.0056 & 0.056 & \pm 0.0028 \\
\hline \hline
\end{tabular}

The assessment of the frequency at which the signal is de-clustered shows that EVT can be applied to the distortion descriptor signal for complex intakes. The EVT model demonstrates a relatively robust technique to estimate extreme levels of distortion independently of the $S t_{d}$ used. However, the number of exceedances strongly affects the confidence intervals for the estimated return values and upper bounds. For the rest of the investigation, the signal is de-clustered with $S t_{d}=1.0$ in order to avoid peak clustering and to retain the maximum number of exceedances for the model.

\subsubsection{EVT for local circumferential distortion}

Although $\overline{\Delta P_{C} / P}$ provides a good indication of the distortion intensity, it is spatially averaged across the 5 rings and can sometimes miss a peak circumferential distortion event that is captured by a single ring only. The effect of local circumferential distortion at the AIP is known to impact engine stability. For example, the ingestion of discrete tightly wound vortices can trigger rotating stall cells [29]. The maximum circumferential distortion descriptor $\left(\Delta P_{C} / P\right)_{\max }$ which has been used to assess the maximum distortion level based on a single ring (section 3.2) can have different characteristics to the $\overline{\Delta P_{C} / P}$ in terms of the unsteady variation and the maximum levels. Within this context, the established EVT method is applied to the de-clustered signal of $\left(\Delta P_{C} / P\right)_{\max }$ for duct $\mathrm{A}$ and $\mathrm{B}$ to highlight the changes in circumferential distortion prediction. Although the signal of $\left(\Delta P_{C} / P\right)_{\max }$ is not necessary correlated from one time step to another, a high level of unsteady total pressure distortion is contained within frequencies ranging from $\mathrm{St}=0.5$ to 1.1 (Fig. 7). Therefore the signal is similarly de-clustered as for the treatment of the $\overline{\Delta P_{C} / P}$ signal with a frequency of $\mathrm{St}=1.0$. The thresholds selected are 0.047 and 0.050 for duct $\mathrm{A}$ and B. The statistics of the two de-clustered signals is different (Fig. 14a, d). Duct A signal is nearly symmetric around the mean with a skewness value of 0.09 . However the excess kurtosis of 0.36 demonstrates that more weight is attributed to the tails than a normal distribution. For Duct B, the de-clustered signal for $\left(\Delta P_{C} / P\right)_{\max }$ is positively skewed with a skewness value of 0.64 . Therefore duct $\mathrm{B}$ has a greater likelihood of the occurrence of an extreme distortion event that affects the distribution with a tendency to increase the weight of the right-hand tail. However, the distribution is also leptokurtic with an excess kurtosis value of 0.66. For Duct A and B, the distribution can be considered close to normal as the excess kurtosis is less than one.

The General Pareto Distributions for duct A and B are significantly different (Fig. 14b, e). This affects the estimation of the return plots (Fig. 15) and the quality of the modelled quantiles (Fig. 14c and f). For duct A, the predicted value $\left(\Delta P_{C} / P\right)_{\max }$ for a return period of $600 \mathrm{~s}\left(P_{600 s}\right)$ is 0.07 which is $45 \%$ higher than $P_{600 s}$ calculated for $\overline{\Delta P_{C} / P}$. For duct $\mathrm{B}, P_{600 s}$ for $\left(\Delta P_{C} / P\right)_{\text {max }}$ is $58 \%$ higher than the value of $P_{600 s}$ estimated for $\overline{\Delta P_{C} / P}$. The mean upper bounds $\left(U_{b}\right)$ estimated for duct $\mathrm{A}$ and $\mathrm{B}$ are 0.081 and 0.099 , respectively, and correspond to the maximum expected levels of local circumferential distortion. 


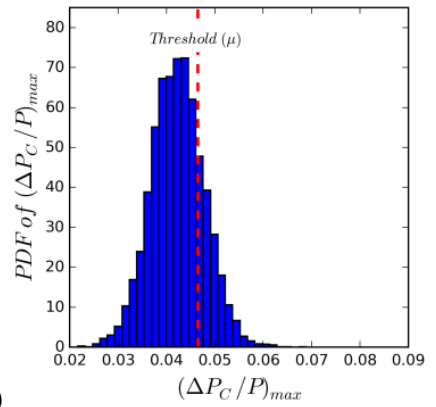

a)

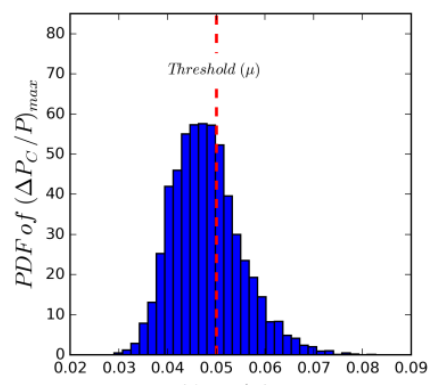

d)

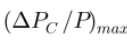

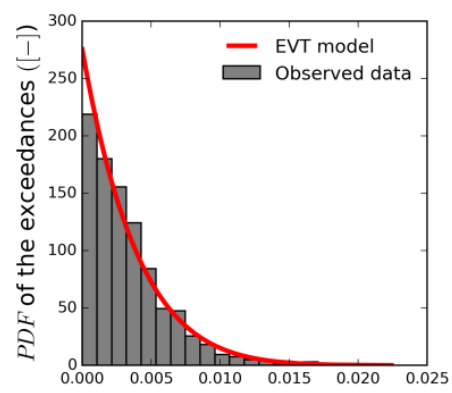

b)

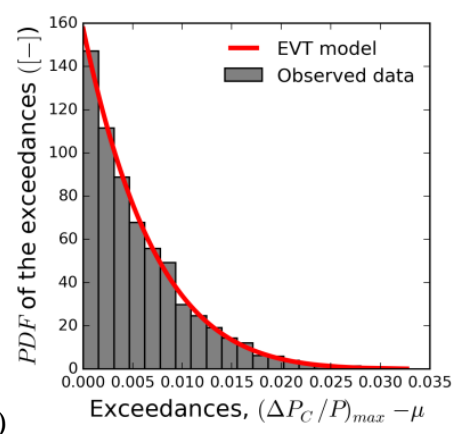

e)

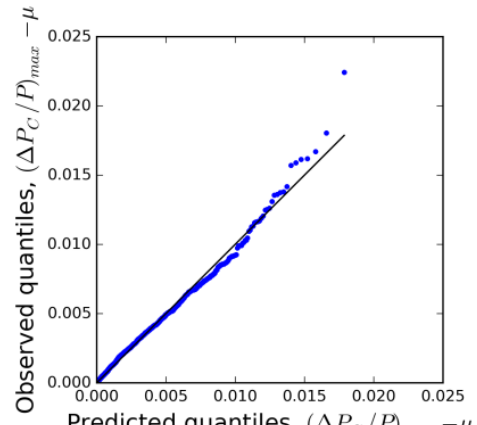

c)

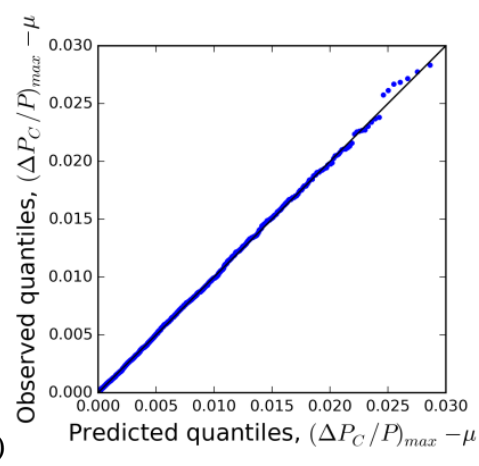

Fig. 14 EVT model diagnosis for duct A (top) and duct B (bottom)

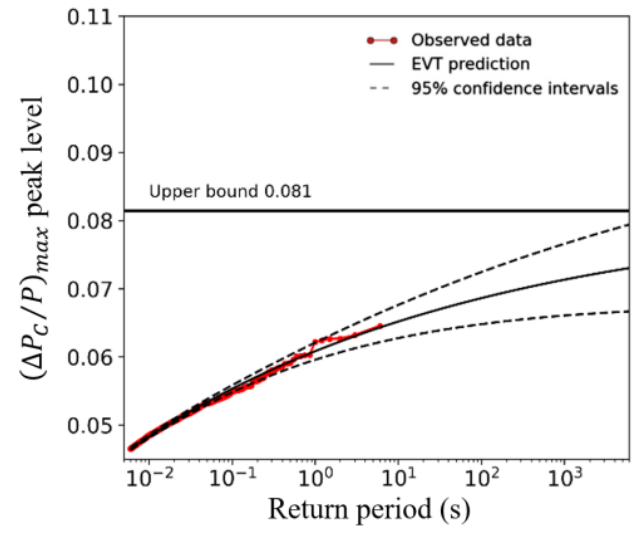

b)

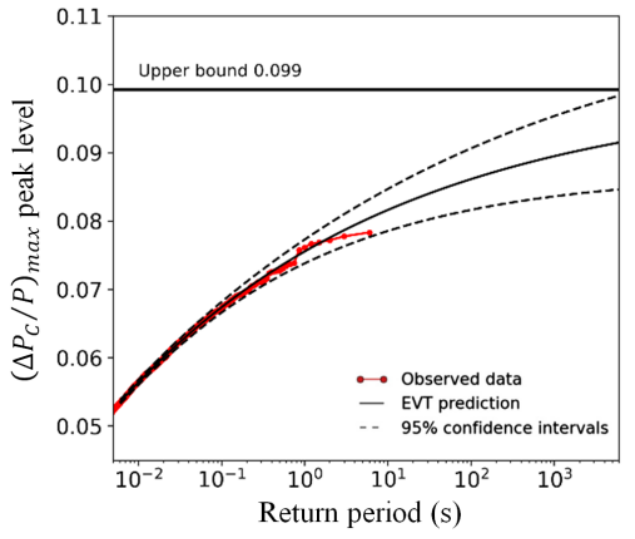

Fig. 15 Return level predictions for a) $\operatorname{duct} A$ and b) duct $B$

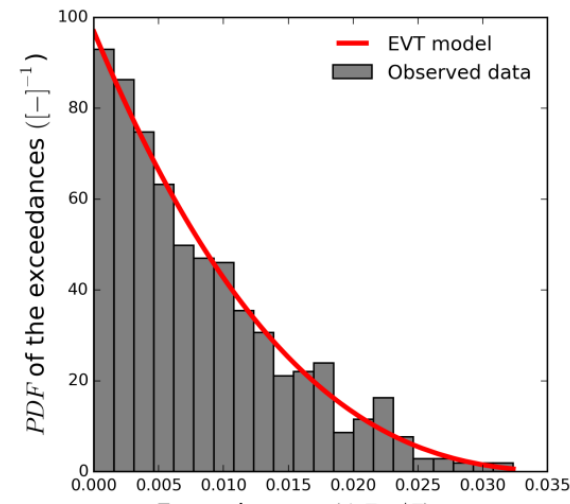

a)

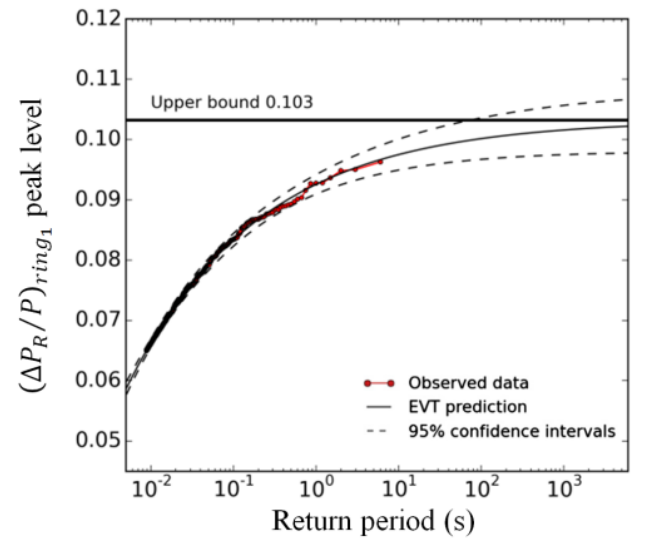

Fig. 16 Comparison between observed and model a) exceedances distribution and b) return plot for $\left(\Delta P_{r} / P\right)_{r i n g}$ 


\subsubsection{EVT for Radial distortion}

In addition to the circumferential distortion, the radial distortion at the AIP can have a substantial negative effect on the compressor stability. Radial distortion strongly affects the blade loading and can also contribute to the reduction in surge margin [4]. While a steady radial distortion may be mitigated by the rotor design, unpredicted peak radial distortion events can increase vibration and compromise the compressor life integrity. Duct B demonstrated large distortion events near the hub caused by the pressure loss region been localised at the centre of the AIP. These events can be identified by the inner ring radial distortion descriptor $\left(\Delta P_{r} / P\right)_{\text {ring }_{1}}$ (Fig. 8) The EVT model is applied on the $\left(\Delta P_{r} / P\right)_{\text {ring }_{1}}$ signal of duct B. The signal is de-clustered with $S t_{d}=1.0$. The thresholds used to assess the peak events for $\left(\Delta P_{r} / P\right)_{\text {ring }_{1}}$ is $\mu=0.065$. The EVT model successfully fits the observed data exceedances distribution (Fig. 16a) and returns the peak value prediction (Fig. 16b). The upper bound estimation $\left(U_{b}=0.103\right)$ is $5.5 \%$ higher than the maximum $\left(\Delta P_{r} / P\right)_{\text {ring }_{1}}$ level observed during the experiment and the return value for a period of 600 seconds is $P_{600 s}=0.101$ with a confidence interval $C I_{P_{600 s}}=0.004$. This indicates that most of the extreme events are captured during the $6 \mathrm{~s}$ of measurements. For comparison, the estimated upper bound for the local maximum circumferential descriptors $\left(\Delta P_{C} / P\right)_{\max }$ was about $20 \%$ higher than the observed maximum peak value for duct B. Furthermore, the shape parameter is significantly lower with $\xi$ equal to -0.12 and -0.27 for $\left(\Delta P_{C} / P\right)_{\max }$ and $\left(\Delta P_{r} / P\right)_{\text {ring }_{1}}$ respectively. As a consequence, the EVT model for $\left(\Delta P_{r} / P\right)_{\text {ring }_{1}}$ converges faster toward the upper bound. Therefore, the EVT application can be extended to local descriptors in order to estimate local peak value for a given acquisition time.

\subsubsection{EVT convergence study}

The convergence assessment of the EVT model has been performed in order to investigate if $2 \mathrm{~s}$ of data is sufficient to predict peak events for a subscale wind tunnel test as reported by Jacocks and Kneile [27]. The assessment is performed over the three distortion descriptors $\overline{\Delta P_{C} / P},\left(\Delta P_{C} / P\right)_{\max }$ and $\left(\Delta P_{r} / P\right)_{\text {ring } 1}$ for duct $\mathrm{B}$. Each signal is modelled with EVT with a sample size ranging from $0.5 \mathrm{~s}$ to $6 \mathrm{~s}$ while keeping the de-clustering frequency constant at $S t_{d}=1.0$. The convergence investigation is performed over the return model calculated from the EVT and their respective confidence intervals non-dimensionalized by the return levels (Fig. 17), the shape parameter $\xi$, the estimation of the upper bound $\left(U_{b}\right)$ and the number of exceedances $k$ (Fig. 18). For the mean circumferential descriptors $\overline{\Delta P_{C} / P}$, the return plot converged monotonically toward the '6s-curve' when the data sample length range from $0.5 \mathrm{~s}$ to $6 \mathrm{~s}$ (Fig. 17a). The return value $P_{100 t}$ for a sample length of $t=5 \mathrm{~s}$ is 0.056 with a $11 \%$ uncertainty estimation (Fig. 17b). The difference from the equivalent return period estimated from the 6 s data set is negligible. The shape factor $\xi$ also monotonically varies as the sample length increases from $0.5 \mathrm{~s}$ to $6 \mathrm{~s}$ and does not significantly change from $5 \mathrm{~s}$ to $6 \mathrm{~s}$. The change in upper bound estimation $\left(U_{b}\right)$ for a signal based on $5 \mathrm{~s}$ and $6 \mathrm{~s}$ is decreased by less than $1 \%$. Therefore the convergence assessment indicates that for the $\overline{\Delta P_{C} / P}$ signal, $5 \mathrm{~s}$ of data is enough to obtain a converged EVT model with a number of exceedances of about 1100 . Furthermore, as the length of the signal is increased, the confidence interval is monotonically reduced (Fig. 17b) which provide a conservative estimate of the return levels for a signal of less than $5 \mathrm{~s}$.

For the maximum ring based circumferential distortion descriptors $\left(\Delta P_{C} / P\right)_{\max }$, the return plots (Fig. 17c) and $\xi$ (Fig. 18a) indicate a non-monotonic convergence. However, for more than $3 \mathrm{~s}$ of data, the changes in return value, $\xi$ and $U_{b}$ are not significant. The value of $P_{300 s}$ estimated for $3 \mathrm{~s}, 5 \mathrm{~s}$ and $6 \mathrm{~s}$ of data is also slightly affected with a change from 0.077 to 0.079 . However, $C I_{P_{300}}$ decreases from about $17 \%$ to $12 \%$ for the EVT model calculated from $3 \mathrm{~s}$ and $6 \mathrm{~s}$ of data, respectively. Therefore, although the return plot shows small variation from $3 \mathrm{~s}$ of data, the evaluation of the confidence intervals does not indicate a fully converged solution at $6 \mathrm{~s}$ of data. This is due to the nature of the signal that contains larger peak distortion events which help to fit the EVT model but increase the variance of the return levels.

The convergence of the radial distortion based on the inner ring $\left(\Delta P_{r} / P\right)_{\text {ring }_{1}}$ is also non-monotonic (Fig. 17e). The assessment of the convergence of the confidence interval (Fig. 17f) shows an almost identical uncertainty prediction for the EVT model based on 5s and 6s. However, the evaluation of the shape parameter $\xi$ does not provide enough evidence that the EVT model is fully converged for a data set of $6 \mathrm{~s}$. Furthermore, the number of exceedance for $6 \mathrm{~s}$ of data is 600 . This is relatively low compared with 1100 for $\overline{\Delta P_{C} / P}$ with $5 \mathrm{~s}$ of data and 850 for $\left(\Delta P_{C} / P\right)_{\max }$ with $3 \mathrm{~s}$ of data (Fig. 18). As for the other descriptors, the upper bound $\left(U_{b}\right)$ estimation does not change significantly (less than 1\%) for the EVT model based on $5 \mathrm{~s}$ and $6 \mathrm{~s}$ of data (Fig. 18).

Therefore, the convergence study indicates that for the mean distortion descriptor $\overline{\Delta P_{C} / P}$ the EVT model reaches convergence with about 5 seconds of data which corresponds to 1100 exceedances. For $\left(\Delta P_{C} / P\right)_{\max }, 3 \mathrm{~s}$ of data with 850 exceedances are enough to estimate a converged solution for the return values and the upper bound. The local inner ring radial descriptor $\left(\Delta P_{r} / P\right)_{\text {ring }_{1}}$ needs more than $6 \mathrm{~s}$ of data to be converged. In general, less than $3 \mathrm{~s}$ of data is not sufficient to provide an accurate estimate with the EVT model.

\section{Conclusions}

An assessment of the unsteady total pressure field at the AIP of two S-ducts showed notable levels of unsteady flow distortion which primarily depends on the duct vertical offset with the main unsteadiness up to frequencies of about $\mathrm{St}=1.5$. The mean circumferential distortion descriptors are relatively insensitive to the change in S-duct offset. The temporal variation of $\overline{\Delta P_{C} / P}$ shows the presence of peak distortion events that deviate from the time averaged flow field. The increase in $\mathrm{S}$-duct offset promotes a greater unsteady variation in the total pressure flow field and the assessment of the local ring based maximum circumferential descriptor 
$\left(\Delta P_{C} / P\right)_{\max }$ facilitates the identification of extreme distortion events. Analysis of the unsteady distortion signatures showed that peak events in circumferential and radial distortion were not synchronous. For both ducts, events with both high radial and circumferential distortion were identified, highlighting the need to evaluate both types of distortion.
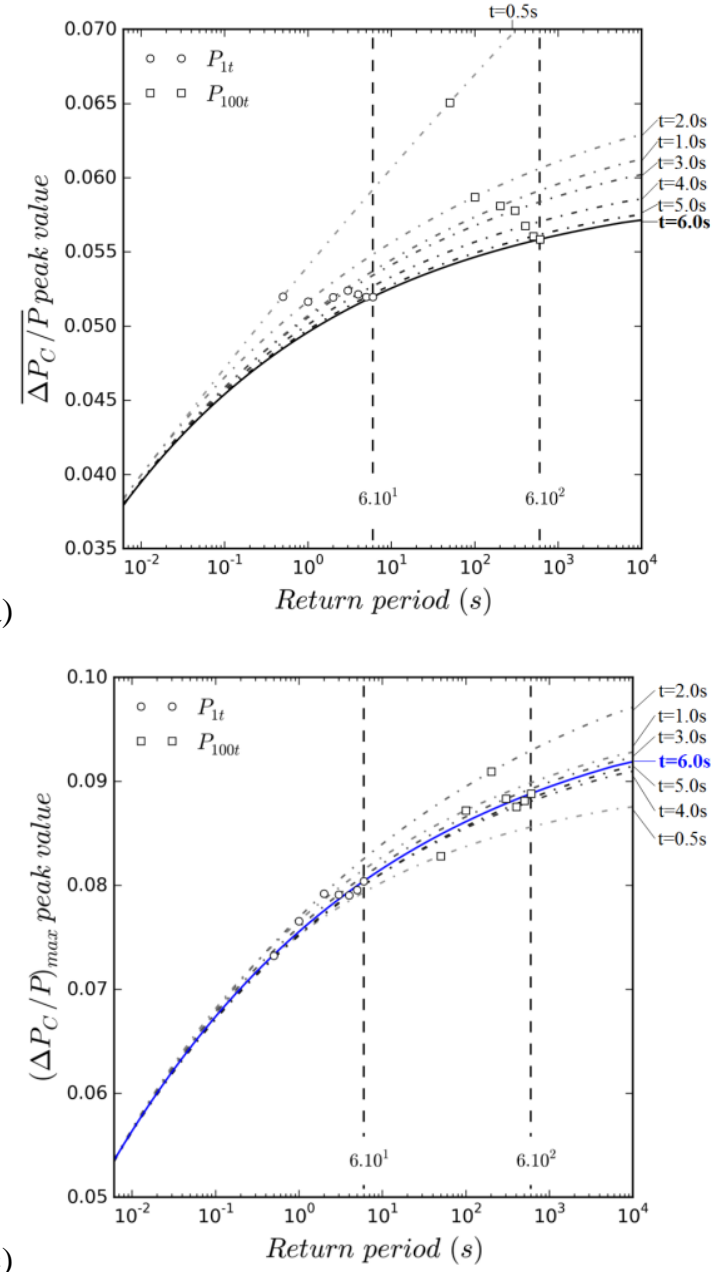

c)

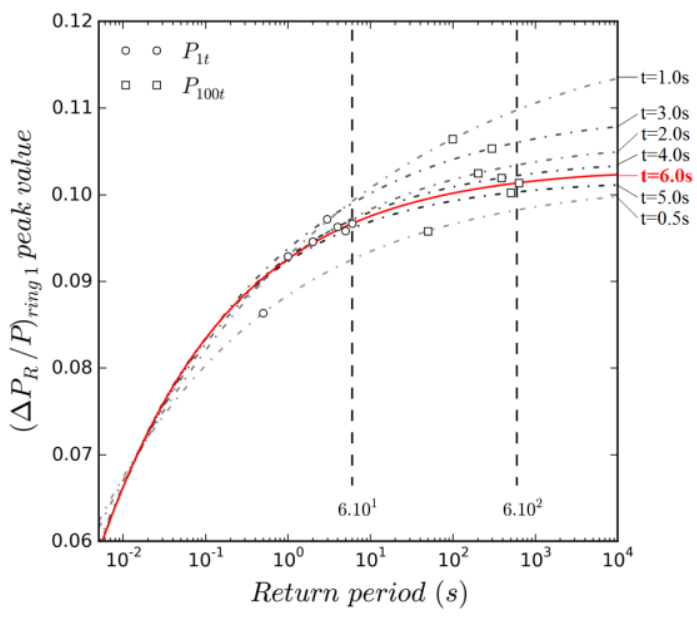

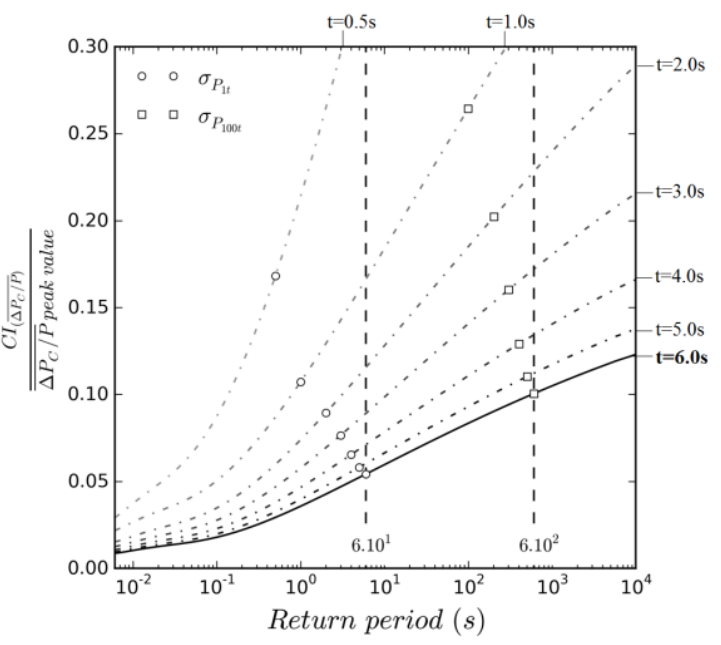

b)

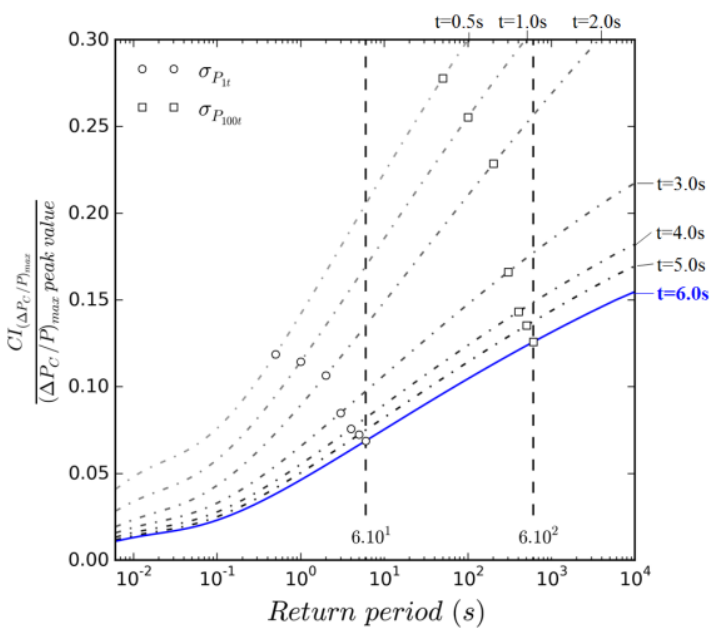

d)

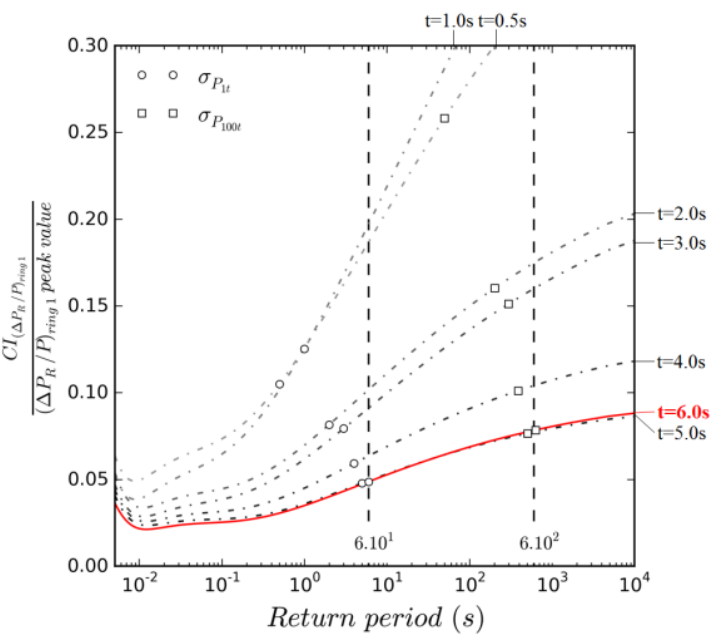

Fig. 17 Convergence study for the EVT return peak value and confidence intervals $C I$ for $\overline{\Delta P_{C} / P}(a, b),\left(\Delta P_{C} / P\right)_{m a x}(c, d)$ and $\left(\Delta P_{r} / P\right)_{\text {ring1 } 1}(e, f)$. 


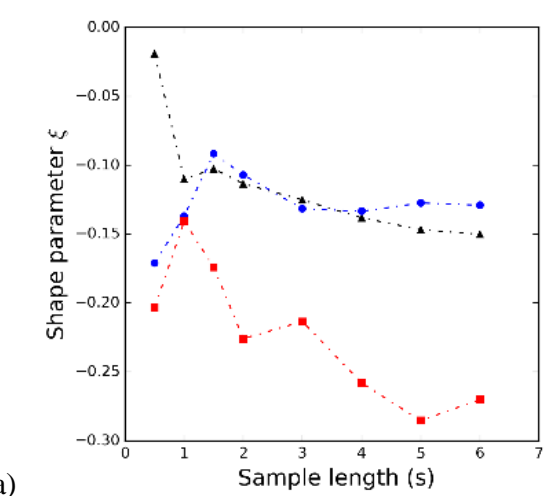

a)

b)

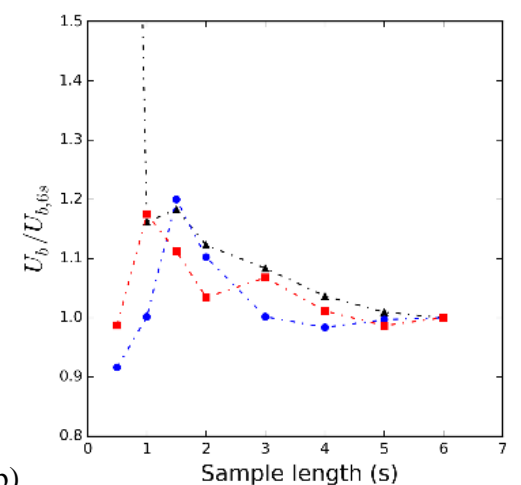

$$
\text { « } \cdots \overline{\Delta P C / P} \quad \cdots \cdot\left(\Delta P_{C} / P\right)_{\max } \quad \cdots \cdots\left(\Delta P_{R} / P\right)_{1}
$$

Fig. 18 Statistical convergence of the EVT

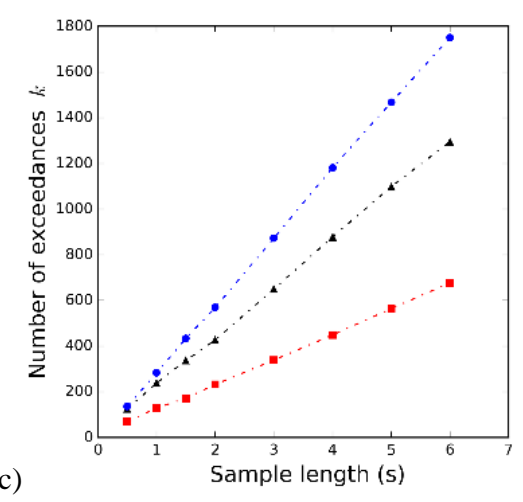

c)

The Extreme Value Theory (EVT) was successfully applied on the unsteady distortion signal for both S-ducts. EVT is used to estimate the peak distortion levels associated with a time duration test of 100 times the observation time available. As the temporal resolution of the distortion signal is several orders of magnitudes higher than the main flow frequencies observed within the flow field, a de-clustering process was applied. This was used to avoid having a single distortion peak captured by more than one temporal measurement which violates an underpinning assumption of the EVT method. The de-clustering frequency assessment showed that the EVT was mainly insensitive to the de-clustering frequency as long as sufficient exceedances were identified for the model. Therefore it is recommended, for this type of flow field, to use a de-clustering frequency slightly greater than the highest characteristic frequency in order to maximise the number of peak observations. The analysis showed that the nominal number of exceedances is sensitive to the type of flow field investigated. A flow field more prone to extreme events, such as the high offset duct, needed a greater number of exceedances in order to provide an accurate prediction of the return value. This is caused by the increased probability of rare events which increase the variance of the EVT parameters and increase the confidence intervals associated to the return values. Overall, an exceedance number of approximately 800 should provide a reasonably converged estimation of the return value and upper bound for the three distortion descriptors investigated in this research.

Previous publications reported that for a typical model scale complex intake, an acquisition time of $2 \mathrm{~s}$ of data is sufficient. A sensitivity assessment of the EVT method with sample duration showed that this is insufficient for complex unsteady flow fields such as those investigated in this research. This study demonstrated that for the spatially averaged mean circumferential distortion descriptor $\overline{\Delta P_{C} / P}$, at least $5 \mathrm{~s}$ of data is needed to reach an error below $10 \%$. For the local ring based descriptors $\left(\Delta P_{C} / P\right)_{\max }$ and $\left(\Delta P_{r} / P\right)_{\text {ring } 1}$, $4 \mathrm{~s}$ of data provides a converged solution of the return plots. More than $6 \mathrm{~s}$ of data is needed to reduce the confidence intervals below $10 \%$ for $\left(\Delta P_{C} / P\right)_{\max }$. Overall, the EVT analysis also shows that the acquisition time required to obtain a converged EVT model increases with the flow unsteadiness and that a minimum of $6 \mathrm{~s}$ is needed for the three distortion descriptors considered in this work for a typical model scale experiment such as the high offset duct B. Therefore, this paper demonstrated that the Extreme Value Theory based on the peak over thresholds methods is a viable tool for unsteady distortion assessment for complex intakes.

\section{References}

[1] D. Bowditch, R. Coltrin, A survey of inlet/engine distortion compatibility, $19^{\text {th }}$ Jt. Propulsion Conf., American Institute of Aeronautics and Astronautics, 1983, doi:10.2514/6.1983-1166

[2] D. Zonars, Dynamic Characteristics of Engine Inlets, AGARD Lecture Series No. 53, Paper No. 6, May 1972

[3] F. Aulehla, Intake Swirl - A Major Disturbance Parameter in Engine/Intake Compatibility, Congr. Int. Counc. Aeronaut. Sci., 1982, ICAS-824.8.1.

[4] Society of Automotive Engineers, Inlet Total Pressure Distortion Considerations for Gas Turbine Engines, SAE Aerospace Information Report 1419C, Warrendale, PA, USA, 2017

[5] D.D. Williams, J.O. Yost, Some aspects of inlet/engine flow compatibility, Aeronaut. J. 77 (1973) 483-492. doi:10.1017/s0001924000041610.

[6] L.M. Wenzel, R.J. Blaha, Analysis of dynamic inlet distortion applied to a parallel compressor model, NASA TM-X-3522, May 1977.

[7] W.T. Cousins, History, philosophy, physics, and future directions of aircraft propulsion system / inlet integration, Proceedings of ASME Turbo Expo 2004, pp. 305-320, Vienna, Austria, June 2004.

[8] P.K. Zachos, D.G. MacManus, D. Gil-Prieto, N. Chiereghin, Flow Distortion Measurements in Convoluted Aero Engine Intakes, AIAA J. Vol. 54, No. 9, Sep. 2016, 2819-2832, doi: 10.2514/1.J054904

[9] D. Gil-Prieto, D.G. MacManus, P.K. Zachos, G. Tanguy, K.R. Menzies, Convoluted intake distortion measurements using stereo particle image velocimetry, AIAA J. Vol. 55, 2017, 1878-1892 doi: 10.2514/1.J055467

[10] R.P.M. Rademakers, S. Bindl, R. Niehuis, Effects of Flow Distortions as They Occur in S-Duct Inlets on the Performance and Stability of a Jet Engine, J. Eng. Gas Turbines Power. Vol. 138, 2016, 022605. doi:10.1115/1.4031305. 
[11] D.G. MacManus, N. Chiereghin, D.G. Prieto, P. Zachos, Complex Aeroengine Intake Ducts and DynamicDistortion, AIAA J. Vol. 55, 2017, 2395-2409, doi:10.2514/1.J054905

[12] N.C. Bissinger, T. Breuer, Basic principles - Gas turbine compatibility - Intake aerodynamics aspects, Encyclopedia of Aerospace Engineering, Dec. 2010.

[13] J. Jacocks, Statistical analysis of distortion factors, 8th Jt. Propuls. Spec. Conf., American Institute of Aeronautics and Astronautics, 1972, doi:10.2514/6.1972-1100.

[14] R. Borg, A Synthesis Inlet Method for Estimating Maximum Instantaneous Distortion Based on Measured Inlet Steady State and RMS Pressures, AGARD-CP-301, Aerodyn. Power Plant Install. 1981.

[15] J.R. Melick H., A. Ybarra, D. Bencze, Estimating maximum instantaneous distortion from inlet total pressure RMS measurements, 14th Jt. Propuls. Conf., American Institute of Aeronautics and Astronautics, 1978. doi:10.2514/6.1978-970.

[16] W.P. Norby, Dynamic Inlet Distortion Prediction with a Combined Computational Fluid Dynamics and Distortion Synthesis Approach, $16^{\text {th }}$ AIAA Applied Aerodynamics Conference, 1998, doi: 10.2514/6.1998- 2735.

[17] D. Sedlock, Improved statistical analysis method for prediction of maximum inlet distortion, 20th Jt. Propuls. Conf., American Institute of Aeronautics and Astronautics, 1984 , doi:10.2514/6.1984-1274.

[18] S. Coles, An Introduction to Statistical Modeling of Extreme Values, 1st ed., Springer-Verlag London, London, 2001.

[19] D. Gil-Prieto, D.G. MacManus, P.K. Zachos, G. Tanguy, F. Wilson, N. Chiereghin, Delayed detached-eddy simulation and particle image velocimetry investigation of S-Duct flow distortion, AIAA J. 55 (2017). doi:10.2514/1.J055468.

[20] L.R. Owens, B.G. Allan, S.A. Gorton, Boundary-Layer-Ingesting Inlet Flow Control, J. Aircr. Vol. 45, 2008 1431-1440. doi:10.2514/1.36989.

[21] A.N. Gissen, B. Vukasinovic, M.L. McMillan, A. Glezer, Distortion management in a boundary layer ingestion inlet diffuser using hybrid flow control, J. Propuls. Power. Vol. 30, 2014 834-844, doi: 10.2514/1.B34981.

[22] A.-L. Delot, E. Garnier, D. Pagan, Flow control in a high-offset subsonic air intake, 47th AIAA/ASME/SAE/ASEE Jt. Propuls. Conf. Exhib. 2011, San Diego, California, doi: 10.2514/6.2011-5569

[23] E. Garnier, Flow Control by Pulsed Jet in a Curved S-Duct: A Spectral Analysis, AIAA J. Vol. 53, 2015, 2813- 2827, doi: 10.2514/1.J053422.

[24] S.R. Wellborn, T.H. Okiishi, B. A. Reichert, A Study of the Compressible Flow though a Diffusing S-Duct, NASA TM 106411, Dec. 1993.

[25] A.-L. Delot, R. Scharnhorst, A Comparison of Several CFD Codes with Experimental Data in a Diffusing S-Duct, 49th AIAA/ASME/SAE/ASEE Jt. Propuls. Conf., American Institute of Aeronautics and Astronautics, 2013, doi:10.2514/6.2013-3796.

[26] D.D. Williams, Review of the Current Knowledge on Engine Response to Distorted Inflow Conditions, AGARD -CP-400, Engine Response to Distorted Inflow Cond. Paper No. 1, Mar 1987.

[27] J. L. Jacocks, K.R. Kneile, Statistical Prediction of Maximum Time-Variant Inlet Distortion Levels, Technical report AEDC TR-74-121, Arnolds Development Center, Tennessee, USA, 1975.

[28] A. Kalpakli Vester, R. Örlü, P.H. Alfredsson, POD analysis of the turbulent flow downstream a mild and sharp bend, Exp. Fluids. Vol. 56, 2015, doi:10.1007/s00348-015-1926-6.

[29] G.A. Mitchell, Effect of Inlet Ingestion of a Wing Tip Vortex on Compressor Face Flow and Turbojet Stall Margin, NASA Technical Report, TM X-3246, 1975.

[30] T.M. Berens, A.-L. Delot, M. Chevalier, J. Van Muijden, R. Waaijer, P. Tattersall, Application of CFD to high offset intake diffusers, Garteur AD/AG-43, October 2012. 
2018-04-24

\section{Characteristics of unsteady total pressure distortion for a complex aero-engine intake duct}

\section{Tanguy, Geoffrey}

\section{Elsevier}

Tanguy G, MacManus DG, Garnier E, Martin PG. (2018) Characteristics of unsteady total pressure distortion for a complex aero-engine intake duct. Aerospace Science and Technology, Volume 78, July 2018, pp. 297-311

https://doi.org/10.1016/j.ast.2018.04.031

Downloaded from Cranfield Library Services E-Repository 